\title{
Exact Bremsstrahlung and effective couplings
}

\author{
Vladimir Mitev ${ }^{a, b}$ and Elli Pomoni ${ }^{c, d}$ \\ ${ }^{a}$ Institut für Physik, WA THEP, Johannes Gutenberg-Universität Mainz, \\ Staudingerweg 7, 55128 Mainz, Germany \\ ${ }^{b}$ Institut für Mathematik und Institut für Physik, Humboldt-Universität zu Berlin, \\ IRIS Haus, Zum Großen Windkanal 6, 12489 Berlin, Germany \\ ${ }^{c}$ DESY Hamburg, Theory Group, \\ Notkestrasse 85, D-22607 Hamburg, Germany \\ ${ }^{d}$ Physics Division, National Technical University of Athens, \\ 15780 Zografou Campus, Athens, Greece \\ E-mail: vmitev@uni-mainz.de, elli.pomoni@desy.de
}

ABSTRACT: We calculate supersymmetric Wilson loops on the ellipsoid for a large class of $\mathcal{N}=2$ SCFT using the localization formula of Hama and Hosomichi. From them we extract the radiation emitted by an accelerating heavy probe quark as well as the entanglement entropy following the recent works of Lewkowycz-Maldacena and Fiol-GerchkovitzKomargodski. Comparing our results with the $\mathcal{N}=4 \mathrm{SYM}$ ones, we obtain interpolating functions $f\left(g^{2}\right)$ such that a given $\mathcal{N}=2$ SCFT observable is obtained by replacing in the corresponding $\mathcal{N}=4 \mathrm{SYM}$ result the coupling constant by $f\left(g^{2}\right)$. These "exact effective couplings" encode the finite, relative renormalization between the $\mathcal{N}=2$ and the $\mathcal{N}=4$ gluon propagator and they interpolate between the weak and the strong coupling. We discuss the range of their applicability.

KeYwords: AdS-CFT Correspondence, Supersymmetric gauge theory, Wilson, 't Hooft and Polyakov loops, Matrix Models

ArXiv EPRINT: 1511.02217 


\section{Contents}

1 Introduction 1

2 Review 3

2.1 The cusp anomalous dimension and the Bremsstrahlung function 3

2.2 The substitution rule in the purely gluonic $\mathrm{SU}(2,1 \mid 2)$ sector 4

$\begin{array}{lll}3 & \text { Wilson loops on ellipsoids } & 6\end{array}$

$\begin{array}{lll}4 & \text { Saddle point approximation } & 7\end{array}$

4.1 Weak coupling results $\quad 9$

$\begin{array}{ll}4.2 \text { Strong coupling results } & 11\end{array}$

5 The Bremsstrahlung function and the entanglement entropy 11

$\begin{array}{ll}5.1 \text { The Bremsstrahlung function } & 12\end{array}$

$\begin{array}{ll}5.2 \text { Entanglement entropy } & 13\end{array}$

6 Universality of the coupling substitution rule 14

$\begin{array}{lll}7 & \text { Conclusions and outlook } & 17\end{array}$

$\begin{array}{lr}\text { A Chebyshev polynomials } & 20\end{array}$

$\begin{array}{ll}\text { B Rewriting the partition functions } & 21\end{array}$

$\begin{array}{ll}\text { C The weak coupling expansion } & 25\end{array}$

$\begin{array}{lr}\text { D The strong coupling limit } & 27\end{array}$

\section{Introduction}

Thanks to its maximal supersymmetry, $\mathcal{N}=4 \mathrm{SYM}$ is the best understood interacting gauge theory in four dimensions. It behooves us to apply our knowledge of $\mathcal{N}=4 \mathrm{SYM}$ to extract results for other gauge theories. We recently observed that some quantities in certain gauge theories can be obtained by a "substitution rule" in which the $\mathcal{N}=4$ gauge coupling is replaced by an effective coupling $[1,2]$. The essential question then arises: are the effective couplings universal, i.e. independent of the observable computed? Addressing this question is the main motivation of this paper.

A powerful tool in the study of $\mathcal{N}=4 \mathrm{SYM}$ is integrability [3]. Integrability was also discovered in the spectral problem of planar $\mathcal{N}=2$ SCFTs, for a purely gluonic subset of local operators with $\mathrm{SU}(2,1 \mid 2)$ symmetry that is closed under renormalization [1]. The 


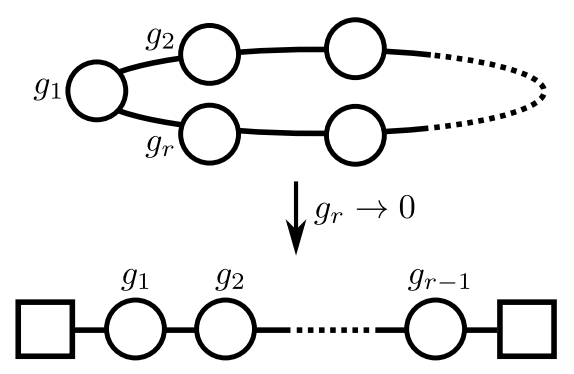

Figure 1. The $\hat{A}_{r-1} \mathcal{N}=2 \mathrm{SCFT}$ elliptic quivers with $\mathrm{SU}(N)^{r}$ color group. Linear quiver theories can be obtained by taking a limit in which one of the gauge couplings $g_{r} \rightarrow 0$. This procedure produces the correct results only in the weak coupling expansion. The strong coupling limit and $g_{r} \rightarrow 0$ do not commute.

mixing matrix of anomalous dimensions of planar $\mathcal{N}=2$ SCFTs is obtained by the $\mathcal{N}=4$ SYM result after replacing the $\mathcal{N}=4$ SYM coupling constant $g^{2}$ by an effective coupling $f\left(g_{i}^{2}\right)$, a function of all the marginal couplings $g_{i}^{2}$ of the $\mathcal{N}=2$ SCFT, computed via localization in [2].

Pestun's work on localization [4] has led to a plethora of exact results for gauge theories in four dimensions with $\mathcal{N} \geq 2$ supersymmetry, see [5] for a review. These include the vacuum expectation values of supersymmetric Wilson loops and 't Hooft Loops $[4,6,7]$ as well as other observables, not immediately given by localization, such as the cusp anomalous dimension, the entanglement entropy ${ }^{1}[8]$ and the quark anti-quark potential [9-11]. ${ }^{2}$

Searching for more observables $O$ to which the coupling substitution rule can be applied, we compute via localization the large $N$ limit of the $b$-deformed BPS Wilson loops of [14] for a large class ${ }^{3}$ of $\mathcal{N}=2$ SCFT, the quiver diagram of which is depicted in figure 1. From them we extract following $[8,19]$ the entanglement entropy and the radiation emitted by an accelerating heavy probe quark. We compare these observables $O$ with their $\mathcal{N}=4 \mathrm{SYM}$ counterparts, extract the effective couplings $f_{O}$ and address the question of universality. Our results interpolate between the weak and the strong coupling. From the weak coupling we can understand the first few terms in the expansion of the localization result using Feynman diagrams, while from the strong coupling the leading term using AdS/CFT.

This paper is structured as follows. We begin in section 2 with a review of the Bremsstrahlung function and the integrability of the purely gluonic sector in $\mathcal{N}=2$ SCFTs. We then overview in section 3 the setup of the circular Wilson loops on the ellipsoid and their computation via localization. We follow up in section 4 with the saddle point approx-

\footnotetext{
${ }^{1}$ The traditional definition of entanglement entropy comes from thermal field theory where the antisymmetric boundary conditions for the fermions break supersymmetry completely.

${ }^{2}$ The Zamolodchikov metric given by the two-point functions of the exactly marginal operators is another very interesting non-BPS observable that can be extracted from exact localization results $[12,13]$.

${ }^{3}$ Obtaining similar results for other $\mathcal{N}=2$ SCFT with a Lagrangian description is straightforward. For theories that do not have a Lagrangian description, localization is not applicable, and thus the road is not completely paved yet. However, we believe that such Wilson loops could be obtained relatively straightforward, by using the results of [15-18] as well as by combining with AGT intuition.
} 


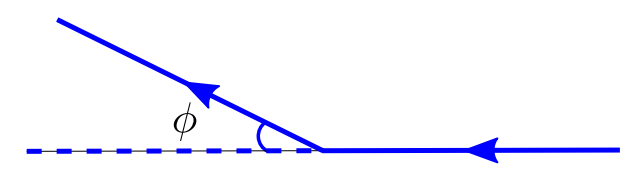

Figure 2. The Wilson line with an Euclidean cusp angle $\phi$.

imation in the planar limit. We discuss the results on Bremsstrahlung function and the entanglement entropy in section 5. The technical aspects of the weak and strong coupling solutions to the saddle point equations are kept in the appendices $\mathrm{C}$ and $\mathrm{D}$. We present an interpretation of some aspects of results, in particular the universality of the coupling substitution in section 6. Finally, we conclude and make some suggestions for future work in section 7 .

\section{Review}

In this section, we present for the convenience of the reader a short review of the main ingredients appearing in this paper. We introduce the cusp anomalous dimension as well as the Bremsstrahlung function. We then explain how to obtain the Bremsstrahlung function in $\mathcal{N}=2$ SCFTs using localization and the work of [19]. Finally, we review our previous work on the coupling substitution rule in the spectral problem of the purely gluonic sector of $\mathcal{N}=2$ SCFTs and show how it can be computed.

\subsection{The cusp anomalous dimension and the Bremsstrahlung function}

The energy emitted by an uniformly accelerating probe quark is proportional to the Bremsstrahlung function $B$

$$
\Delta E=2 \pi B \int d t \dot{v}^{2},
$$

for small velocities $v$. It is well known, see for example [20] for an old review and [21] for a more recent presentation, that $B$ can be obtained from a Wilson line that makes a sudden turn by an angle $\phi$, see figure 2 , at a single specific point that we refer to as the cusp. As discussed already in [22], the vacuum expectation value of such a Wilson loop is both UV and IR divergent with divergences of the form

$$
\left\langle W_{\varphi}\right\rangle \sim e^{-\Gamma_{\text {cusp }}(\varphi) \log \frac{\Lambda_{\mathrm{UV}}}{\Lambda_{\mathrm{IR}}}},
$$

where $\Lambda_{\mathrm{UV}}$, respectively $\Lambda_{\mathrm{IR}}$ is the UV, respectively IR cutoff. In (2.2), we have analytically continued to Minkowski signature by setting $\phi=i \varphi$.

The first important observation for this Wilson loop is the fact that, for large Euclidean angles $\phi \sim \pi$, its cusp anomalous dimension leads to the quark-antiquark potential, while for large values of the Minkowski angle $\varphi$, it grows linearly with $\varphi$ with a slope equal to the light-like cusp anomalous dimension $K$, i.e.

$$
\Gamma_{\text {cusp }}(\varphi) \sim K \varphi
$$


The number $K$, determines the leading logarithmic behavior of the anomalous dimensions of finite twist operators in the large spin limit [23] as

$$
\Delta-S \sim K \log (S) .
$$

The light-like cusp anomalous dimension has been calculated for $\mathcal{N}=4 \mathrm{SYM}$ to four loops [24-26], also using integrability [27], see [28] for a review, and lately even using resurgence techniques $[29,30]$.

The second important observation is that, for small $\varphi$, the divergence of the Wilson loop becomes quadratic in $\varphi$ with coefficient given by the Bremsstrahlung function

$$
\Gamma_{\text {cusp }}(\varphi)=B \varphi^{2}+\mathcal{O}\left(\varphi^{4}\right) .
$$

Importantly, the Bremsstrahlung function can be obtained from other geometries that allow us to compute it via localization. In [21], it was argued that for $\mathcal{N}=4 \mathrm{SYM}, B$ can be obtained from the Wilson loop expectation value on the sphere as $B=\frac{\lambda}{2 \pi} \partial_{\lambda} \log \langle W\rangle$. Furthermore, according to a conjecture by [19], the Bremsstrahlung function of $\mathcal{N}=2$ theories should be given by

$$
B= \pm \frac{1}{4 \pi^{2}} \frac{d}{d b} \log \left\langle W^{ \pm}(b)\right\rangle_{\mid b=1},
$$

where $\left\langle W^{ \pm}(b)\right\rangle$ are the Wilson loop expectation values of circular loops on the ellipsoid with parameter $b$, see (3.5). This formula is true for $\mathcal{N}=4$ SYM [8] but needs to be subjected to further checks or to be derived rigorously for $\mathcal{N}=2$ theories. Formula (2.6) is an input of our paper. However, our study provides consistency checks of (2.6) by verifying that it is compatible with the coupling substitution rule, both in the weak and in the strong coupling limit.

\subsection{The substitution rule in the purely gluonic $\mathrm{SU}(2,1 \mid 2)$ sector}

In $[1],{ }^{5}$ integrability was discovered in the spectral problem of planar $\mathcal{N}=2$ SCFTs for a purely gluonic subset of local operators, closed under renormalization. This set of operators is made out of the fields $\phi, \lambda_{+}^{\mathcal{I}}, \mathcal{D}_{+\dot{\alpha}}$ in one of the $\mathcal{N}=2$ vector multiplets of the theory and transforms under the action of a $\mathrm{SU}(2,1 \mid 2)$ global symmetry. The mixing matrix of anomalous dimensions ${ }^{6}$ of planar $\mathcal{N}=2$ SCFTs is obtained by the $\mathcal{N}=4$ SYM result after replacing the $\mathcal{N}=4 \mathrm{SYM}$ coupling constant as $g^{2} \rightarrow f\left(g_{i}^{2}\right)$, with $f$ a function of all the marginal couplings $g_{i}^{2}$ of the $\mathcal{N}=2$ SCFT. To be more precise, there is a purely gluonic sector together with its respective effective coupling $f_{k}\left(g_{1}^{2}, \ldots, g_{r}^{2}\right)$ for each vector multiplet $V_{k}$ of the theory, but for the $A$-type quivers that we study, the effective couplings are all related by permutations of the marginal couplings. In particular, the light-like

\footnotetext{
${ }^{4}$ Here and elsewhere, we use the following definition of the couplings $\lambda=N g_{\mathrm{YM}}^{2}=(4 \pi g)^{2}$.

${ }^{5}$ See [31-38] for work on which it was based.

${ }^{6}$ The planar limit is essential at this stage for two reasons. Firstly, in order to use the integrability of $\mathcal{N}=4 \mathrm{SYM}$, we have to go to the planar limit. Secondly, the inheritance theorems of [39, 40], according to which the correlation function in the untwisted sector are equal to the $\mathcal{N}=4$ ones at the orbifold point, hold only in the planar limit.
} 
cusp anomalous dimension $K$ is an observable in the purely gluonic sector. As we already mentioned above, $K$ is the leading logarithmic behavior of the anomalous dimensions of finite twist $\Delta-S$ operators in the large spin limit (2.4). Thus, it is in the purely gluonic $\mathrm{SU}(2,1 \mid 2)$ sector, since the twist $\Delta-S$ operators are in the sector. Thus, for $\mathcal{N}=2$ SCFTs the light-like cusp anomalous dimension $K_{\mathcal{N}=2, k}$ for the $k^{\text {th }}$ gauge group is simply given by the $\mathcal{N}=4$ results by the substitution

$$
K_{\mathcal{N}=2, k}\left(g_{i}^{2}\right) \equiv K_{k}\left(g_{i}^{2}\right)=K_{\mathcal{N}=4}\left(f_{k}\left(g_{i}^{2}\right)\right) .
$$

From the Feynman diagrams (weak coupling) point of view, the effective couplings $f_{k}\left(g_{i}^{2}\right)$ is the relative finite renormalization of the $\mathcal{N}=2$ gluon propagator of the $k^{\text {th }}$ color group [2],

$$
f_{k}\left(g_{1}^{2}, \ldots, g_{r}^{2}\right)=g_{k}^{2}+g_{k}^{2}\left[\left(\mathcal{Z}_{g_{k}}^{\mathcal{N}=2}\right)^{2}-\left(\mathcal{Z}_{g_{k}}^{\mathcal{N}=4}\right)^{2}\right] .
$$

and as such depends on all the marginal couplings $g_{i}^{2}$ of the $\mathcal{N}=2$ SCFT. In [2], we computed using Feynman diagrams the relative finite renormalization of the $\mathcal{N}=2$ gluon propagator to three-loops and found that

$$
\begin{aligned}
f_{k}\left(g_{1}^{2}, \ldots, g_{r}^{2}\right)= & g_{k}^{2}+6 \zeta(3) g_{k}^{4}\left[g_{k-1}^{2}+g_{k+1}^{2}-2 g_{k}^{2}\right] \\
& -20 \zeta(5) g_{k}^{4}\left[g_{k-1}^{4}+g_{k+1}^{4}-6 g_{k}^{4}+2 g_{k}^{2}\left(g_{k-1}^{2}+g_{k+1}^{2}\right)\right] .
\end{aligned}
$$

From the strong coupling point of view and using AdS/CFT correspondence, ${ }^{7}$ the effective coupling computes the relation (via the AdS/CFT dictionary) between the effective tension of the string and the coupling constant of the $\mathcal{N}=2$ SCFT

$$
T_{\mathrm{eff}}^{2}=\frac{R^{4}}{\left(2 \pi \alpha^{\prime}\right)^{2}}=f\left(g_{i}^{2}\right) .
$$

Using the AdS/CFT dictionary and the work of [33, 38, 41] in [2], we obtained the leading term of the effective tension of the string at strong coupling and found that it is

$$
f_{k}\left(g_{i}^{2}\right)=r \frac{g_{1}^{2} \cdots g_{r}^{2}}{\sum_{i=1}^{r} \prod_{j \neq i} g_{j}^{2}}+\cdots
$$

On the string theory side, the observables of the purely gluonic SU $(2,1 \mid 2)$ sector correspond to string states classically living in the $A d S_{5} \times S^{1}$ factor $^{8}$ of the geometry with the $S^{1}$ corresponding to the $\mathrm{U}(1)_{r}$ of the $\mathcal{N}=2$ theories. The chiral $\operatorname{Tr}\left(\phi^{\ell}\right)$ with $\Delta=r$ are charged under the $\mathrm{U}(1)_{r}$ and correspond to sugra KK reduction modes on this $S^{1}$. See [33] and also [42] for a recent discussion.

In [2] the effective couplings were also extracted from the circular Wilson loop expectation value on $S^{4}$, calculated thanks to localization [4]. The results agree with (2.9) and (2.11). It now is vital to study as many observables as possible in order to check the extend to which the effective couplings are universal.

\footnotetext{
${ }^{7}$ Note that the quivers that we are considering have a gravity dual description.

${ }^{8}$ Specifically, the geometry does not factorize, but has an $\mathrm{U}(1)$ isometry.
} 


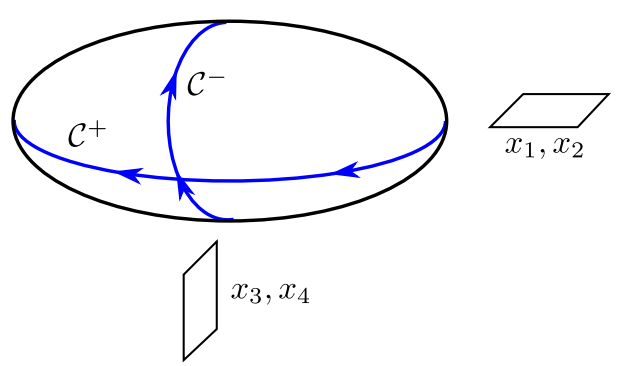

Figure 3. We sketch here two circular Wilson loops $\mathcal{C}^{ \pm}$that can be computed on the ellipsoid via localization. The 4D ellipsoid itself should be understood as a fibration of the 3D ellipsoid over the interval $x_{0} \in[-r, r]$.

A short comment about notation is due. Since we will often be comparing $\mathcal{N}=4$ quantities to $\mathcal{N}=2$ ones, we need to be painstakingly clear about denoting them properly. In general $\mathcal{N}=4$ quantities will be denoted as such, for example $\left\langle W_{\mathcal{N}=4}\right\rangle$ for the vacuum expectation value of the Wilson loop on the sphere. We will specifically be considering the $\mathbb{Z}_{r}$ cyclic quiver $\mathcal{N}=2$ theories, see figure 1 . They have $r$ gauge groups and we will designate the corresponding quantities, such as the Wilson loop expectation values or the Bremsstrahlung functions, simply by labeling them by an index $k \in\{1, \ldots, r\}$, for example $B_{k}$. Furthermore, for the sake of brevity, we shall often abbreviate the dependence of a function $f_{k}\left(g_{1}^{2}, \ldots, g_{r}^{2}\right)$ of all the couplings as $f_{k}\left(g_{i}^{2}\right)$.

\section{$3 \quad$ Wilson loops on ellipsoids}

The 4D ellipsoid is defined, in embedding coordinates, via the equation

$$
\frac{x_{0}^{2}}{r^{2}}+\frac{x_{1}^{2}+x_{2}^{2}}{\ell^{2}}+\frac{x_{3}^{2}+x_{4}^{2}}{\tilde{\ell}^{2}}=1 .
$$

We are interested in the Maldacena-Wilson loops on the ellipsoid

$$
\left\langle W_{k}\left(\mathcal{C}^{ \pm}\right)\right\rangle \equiv\left\langle W_{k}^{ \pm}\right\rangle=\left\langle\frac{1}{N} \operatorname{Tr}_{\square} \operatorname{Pexp} \oint_{\mathcal{C}^{ \pm}} d s\left(i A_{\mu}^{(k)}(x) \dot{x}^{\mu}+\phi^{(k)}(x)|\dot{x}|\right)\right\rangle,
$$

where $\square$ denotes the fundamental representation and $\mathcal{C}^{ \pm}$are the two circular loop located depicted in figure 3. In our case, we have $r$ gauge groups and the index $k$ labels the adjoint scalar $\phi^{(k)}$ and the gauge field $A_{\mu}^{(k)}$ in the vector multiplet of the $k$-th gauge group.

We define the deformation parameter $b$ as

$$
b:=\sqrt{\ell / \tilde{\ell}}
$$

The case $b=1$ corresponds to the sphere $S^{4}$, in which case the localization result was already given by [4]. In [14], the following expression for the partition function (written for simplicity here for a single SUN gauge group) was given

$$
Z=\int d \hat{a} e^{-\frac{8 \pi^{2}}{g_{\mathrm{YM}}^{2}} \operatorname{Tr}\left(\hat{a}^{2}\right)} Z_{1 \text {-loop }}(a, b)\left|Z_{\text {inst }}(a, b)\right|^{2},
$$


where $\hat{a}:=\sqrt{\ell \hat{\ell}} a$. The matrix $a=\operatorname{diag}\left(a_{1}, \ldots, a_{N}\right)$ is subject to the condition $\sum_{i=1}^{N} a_{i}=0$ and is an element of the Cartan subalgebra of $\mathfrak{s u}(N)$. The two Wilson loops that we are able to compute are drawn in figure 3 and are given by

$$
\left\langle W^{ \pm}(b)\right\rangle=\frac{1}{Z} \int d \hat{a} \operatorname{Tr}\left(e^{-2 \pi b^{ \pm 1} \hat{a}}\right) e^{-\frac{8 \pi^{2}}{g_{\mathrm{YM}}^{2}} \operatorname{Tr}\left(\hat{a}^{2}\right)} Z_{1 \text {-loop }}\left|Z_{\text {inst }}\right|^{2}
$$

The perturbative part $Z_{1 \text {-loop }}$ of (3.4) is given by a product over the vector multiplet and hypermultiplet contributions (here only in the bifundamental representation of two SUN gauge groups) with

$$
\begin{aligned}
& Z_{1 \text {-loop }}^{\text {vect }}=\prod_{i<j=1}^{N} \Upsilon\left(i \sqrt{\ell \tilde{\ell}}\left(a_{i}-a_{j}\right) ; b\right) \Upsilon\left(-i \sqrt{\ell \tilde{\ell}}\left(a_{i}-a_{j}\right) ; b\right) \\
& Z_{1 \text {-loop }}^{\text {hyper }}=\prod_{i, j=1}^{N} \Upsilon\left(i \sqrt{\ell \tilde{\ell}}\left(a_{i}^{(1)}-a_{j}^{(2)}\right)+Q / 2 ; b\right)^{-1},
\end{aligned}
$$

where $Q=b+b^{-1}$ and $\Upsilon$ is defined in appendix $\mathrm{C}$ of [17]. We remark that both $Z_{1-\text { loop }}^{\text {vect }}$ and $Z_{1-\text { loop }}^{\text {hyper }}$ are invariant under the transformation $b \leftrightarrow b^{-1}$.

For massless theories, we can rescale the integration variable $a$ and get rid of the factor of $\sqrt{\ell \hat{\ell}}$, whereas for massive one, the factor of $\sqrt{\ell \hat{\ell}}$ is part of the ambiguity of the mass. Rearranging some factors in the special functions, leads us to the expression, proven in appendix B,

$$
Z=\int d a e^{-\frac{N}{2 g^{2}} \sum_{i=1}^{N} a_{i}^{2}} Z_{1 \text {-loop }}(a, b)\left|Z_{\text {inst }}(a, b)\right|^{2},
$$

with $g$ given through the relations $\lambda=N g_{\mathrm{YM}}^{2}=(4 \pi g)^{2}$ and where now the 1-loop part is given by

$$
Z_{1-\text { loop }}^{\text {vect }}=\prod_{i<j=1}^{N}\left(a_{i}-a_{j}\right)^{2} \prod_{i, j=1}^{N} H_{v}\left(a_{i}-a_{j} ; b\right), \quad Z_{1 \text {-loop }}^{\text {hyper }}=\prod_{i, j=1}^{N} H_{h}\left(a_{i}^{(1)}-a_{j}^{(2)} ; b\right)^{-1},
$$

with the functions $H_{v}(x ; b)$ and $H_{h}(x ; b)$ defined in (B.6). These functions have the advantage of being simpler to work with for the weak coupling expansion since they are even in $x$, invariant under $b \leftrightarrow b^{-1}$ and normalized as $H_{v}(0 ; b)=H_{h}(0 ; b)=1$.

\section{Saddle point approximation}

We only consider the $A$ class of $\mathcal{N}=2$ SCFTs, i.e. the $\mathbb{Z}_{r}$ cyclic quivers or the linear quivers. ${ }^{9}$ We shall concentrate on the cyclic, or class $\hat{A}_{r-1}$, of quiver theories, since in the weak coupling limit we can recover the results for the class $A_{r-1}$ linear quivers by taking a limit, see figure 1 . The partition function is can then be written as

$$
\begin{aligned}
Z & =\int \prod_{k=1}^{r} d^{N-1} a^{(k)} \prod_{i<j=1}^{N}\left(a_{i}^{(k)}-a_{j}^{(k)}\right)^{2} e^{-\frac{N}{2 g_{k}^{2}} \sum_{i=1}^{N}\left(a_{i}^{(k)}\right)^{2}} Z_{1-\text { loop }}\left|Z_{\text {inst }}\right|^{2} \\
& =\int \prod_{k=1}^{r} d^{N-1} a^{(k)} e^{-N \mathcal{S}_{\text {eff }}},
\end{aligned}
$$

\footnotetext{
${ }^{9}$ Similar calculations can be found in [43-46], and for the $\mathcal{N}=2^{*}$ theory on the ellipsoid in [47].
} 
where the instanton part $Z_{\text {inst }}$ will be ignored, since we are going to perform a planar limit ${ }^{10}$ computation. We remind that, since we are dealing with $\mathrm{SU}(N)$ gauge groups, the Coulomb parameters satisfy the equation $\sum_{i=1}^{N} a_{i}^{(k)}=0, \forall k=1, \ldots, r$. In the large $N$ limit that we are interested in, we can safely ignore the instanton part [43], and the effective action is given by

$$
\mathcal{S}_{\text {eff }}=\sum_{k=1}^{r}\left[\sum_{i=1}^{N} \frac{1}{2 g_{k}^{2}}\left(a_{i}^{(k)}\right)^{2}-\frac{1}{N} \sum_{i<j=1}^{N} \ln \left(a_{i}^{(k)}-a_{j}^{(k)}\right)^{2}\right]-\frac{1}{N} \ln \left(Z_{1 \text {-loop }}\right) .
$$

For the $\hat{A}_{r-1}$ quivers, the perturbative part of the partition function can be written as

$$
\ln \left(Z_{1 \text {-loop }}\right)=\sum_{k, l=1}^{r} \sum_{i, j=1}^{N}\left[\delta_{k l} \log H_{v}\left(a_{i}^{(k)}-a_{j}^{(l)}\right)-\frac{\delta_{k, l+1}+\delta_{k, l-1}}{2} \log H_{h}\left(a_{i}^{(k)}-a_{j}^{(l)}\right)\right] .
$$

Solving the matrix model in the planar limit is done by considering the saddle point approximation. Specifically, we are computing a vacuum expectation value of some quantity $W$ in the large $N$ limit

$$
\langle W\rangle=\frac{\int \prod_{k=1}^{r} d^{N-1} a^{(k)} W\left(a^{(\ell)}\right) e^{-N \mathcal{S}_{\mathrm{eff}}}}{\int \prod_{k=1}^{r} d^{N-1} a^{(k)} e^{-N \mathcal{S}_{\mathrm{eff}}}}
$$

where by abuse of notation $W$ is also a function of the eigenvalues $a^{(k)}=\left(a_{1}^{(k)}, \ldots, a_{N}^{(k)}\right)$ of the $k^{\text {th }}$ gauge group. Let the effective action have an extremum $\left(\left.\frac{\partial \mathcal{S}_{\text {eff }}}{\partial a}\right|_{a=b}=0\right)$ at $a=\left(a^{(1)}, \ldots, a^{(r)}\right) \equiv b$ and expand the integral $a=b+\frac{1}{\sqrt{N}} x$, we get

$$
\begin{aligned}
\int d a W(a) e^{-N \mathcal{S}_{\mathrm{eff}}}= & \frac{e^{-N \mathcal{S}_{\mathrm{eff}}(b)}}{N^{\text {power }}} \int d x e^{-\frac{1}{2}\left(\mathcal{S}_{\mathrm{eff}}\right), i j(b) x_{i} x_{j}}[W(b) \\
& \left.+\frac{1}{N^{\frac{1}{2}}}\left(W_{, i}(b) x_{i}-\frac{1}{6} W(b)\left(\mathcal{S}_{\mathrm{eff}}\right)_{, i j k} x_{i} x_{j} x_{k}\right)+\cdots\right],
\end{aligned}
$$

where "power" is a number that will drop out in the end and we have used the shorthand $W_{, i j k \cdots}=\partial_{i} \partial_{j} \partial_{k} \cdots W$. It follows that the leading term in the planar limit is given by the function evaluated at the saddle point, i.e.

$$
\langle W(a)\rangle=W(b)+\mathcal{O}(1 / N) .
$$

In our case, the saddle point equations $\partial \mathcal{S}_{\mathrm{eff}} / \partial a_{i}^{(k)}=0$ imply that for all $i=1, \ldots, N$ and all $k=1, \ldots, r$, we must have

$$
\frac{a_{i}^{(k)}}{2 g_{k}^{2}}=\frac{1}{N} \sum_{j \neq i} \frac{1}{a_{i}^{(k)}-a_{j}^{(k)}}-\frac{1}{N} \sum_{l=1}^{r} \sum_{j=1}^{N}\left[\delta_{k l} K_{v}\left(a_{i}^{(k)}-a_{j}^{(l)}\right)-\frac{\delta_{k, l+1}+\delta_{k, l-1}}{2} K_{h}\left(a_{i}^{(k)}-a_{j}^{(l)}\right)\right] .
$$

\footnotetext{
${ }^{10}$ See [43] and [44] for a discussion on this subject.
} 
where $K_{v}$ and $K_{h}$ are defined in (B.7). In the $N \rightarrow \infty$ limit, we replace the eigenvalues $a_{i}^{(k)}$ by normalized densities that are localized on a symmetric interval $\left[-\mu_{k}, \mu_{k}\right]$

$$
\rho_{k}(x)=\frac{1}{N} \sum_{i=1}^{N} \delta\left(x-a_{i}^{(k)}\right), \quad \int_{-\mu_{k}}^{\mu_{k}} \rho_{k}(x) d x=1
$$

which transforms the saddle point equations (4.7) into integral equations. Specifically, we obtain the following system of coupled integral equations:

$$
\frac{x}{2 g_{k}^{2}}=f_{-\mu_{k}}^{\mu_{k}} d y \frac{\rho_{k}(y)}{x-y}-\sum_{l=1}^{r} \int_{-\mu_{l}}^{\mu_{l}}\left[\delta_{k l} K_{v}(x-y)-\frac{\delta_{k, l+1}+\delta_{k, l-1}}{2} K_{h}(x-y)\right] \rho_{l}(y) d y
$$

for $k=1, \ldots, r$. For numerical approximations at small values of the 't Hooft couplings, it is sometimes helpful to rewrite (4.9) by inverting the Hilbert kernel, i.e. by acting with $f_{-\mu_{k}}^{\mu_{k}} \frac{d x}{\sqrt{\mu_{k}^{2}-x^{2}}} \frac{1}{z-x}$ on both sides of the equation. Using (A.2) and (A.5) we get the set of equations

$$
\begin{aligned}
\rho_{k}(x)= & \frac{1}{2 \pi g_{k}^{2}} \sqrt{\mu_{k}^{2}-x^{2}}-\frac{1}{\pi^{2}} f_{-\mu_{k}}^{\mu_{k}} \frac{d y}{x-y} \sqrt{\frac{\mu_{k}^{2}-x^{2}}{\mu_{k}^{2}-y^{2}}} \sum_{l=1}^{r} \int_{-\mu_{l}}^{\mu_{l}} \rho_{l}(z) d z\left[\delta_{k l} K_{v}(y-z)\right. \\
& \left.-\frac{\delta_{k, l+1}+\delta_{k, l-1}}{2} K_{h}(y-z)\right],
\end{aligned}
$$

subject to the normalization condition for the densities

$$
1=\frac{\mu_{k}^{2}}{4 g_{k}^{2}}+\frac{1}{\pi} \int_{-\mu_{k}}^{\mu_{k}} \frac{d y y}{\sqrt{\mu_{k}^{2}-y^{2}}} \sum_{l=1}^{r} \int_{-\mu_{l}}^{\mu_{l}} \rho_{l}(z) d z\left[\delta_{k l} K_{v}(y-z)-\frac{\delta_{k, l+1}+\delta_{k, l-1}}{2} K_{h}(y-z)\right] .
$$

Thanks to (4.6), having obtained the densities by solving the saddle point equations (4.10), we can compute the Wilson loop expectation values for the $k$-th gauge group by plugging the densities

$$
W_{k}^{ \pm}\left(g_{1}, \ldots, g_{r} ; b\right)=\left\langle\frac{1}{N} \sum_{i=1}^{N} e^{-2 \pi a_{i}^{(k)} b^{ \pm 1}}\right\rangle=\int_{-\mu_{k}}^{\mu_{k}} \rho_{k}(x) e^{-2 \pi x b^{ \pm 1}} d x .
$$

\subsection{Weak coupling results}

By weak coupling, we understand the regime for which all the couplings are small, i.e.

$$
g_{i}^{2}=t \kappa_{i}
$$

with the coefficients $\kappa_{i}$ being order one constants and $t \ll 1$. Appendix $\mathrm{C}$ contains further details on the weak coupling expansion of the Wilson loops. From the vacuum expectation values of the Wilson loops on the ellipsoids, we define the "full" effective couplings $f_{k}\left(g_{i}^{2} ; b\right)$ via

$$
\left\langle W_{\mathcal{N}=4}^{+}\left(f_{k}\left(g_{1}^{2}, \ldots, g_{r}^{2} ; b\right) ; b\right)\right\rangle=\left\langle W_{k}^{+}\left(g_{1}^{2}, \ldots, g_{r}^{2} ; b\right)\right\rangle
$$


We could have just as easily used the other Wilson loop $\left\langle W^{-}\right\rangle$in the above. The corresponding effective coupling is simply $f_{k}\left(g_{i}^{2}, b^{-1}\right)$. It is useful to expand these effective couplings in power of $(b-1)$ around $b=1$. We define the coefficients of this expansion as

$$
f_{k}\left(g_{1}^{2}, \ldots, g_{r}^{2} ; b\right):=\sum_{n=0}^{\infty} f_{k}^{(n)}\left(g_{1}^{2}, \ldots, g_{r}^{2}\right)(b-1)^{n} .
$$

In appendix $\mathrm{C}$, we write explicitly the linear equations that need to be solved to obtain the Wilson loop expectation values on the ellipsoids. From the result (C.12) for the $\mathbb{Z}_{2}$ quiver, we get

$$
\begin{aligned}
f_{1}^{(0)}\left(g_{1}^{2}, g_{2}^{2}\right)= & g_{1}^{2}+\left(g_{2}^{2}-g_{1}^{2}\right)\left\{12 \zeta(3) g_{1}^{4}-40 \zeta(5) g_{1}^{4}\left[3 g_{1}^{2}+g_{2}^{2}\right]\right. \\
& -\frac{4}{3} g_{1}^{4}\left[10 \pi^{2} \zeta(5) g_{1}^{4}+108 \zeta(3)^{2}\left(2 g_{1}^{4}-g_{2}^{2} g_{1}^{2}+g_{2}^{4}\right)-105 \zeta(7)\left(8 g_{1}^{4}+5 g_{2}^{2} g_{1}^{2}+g_{2}^{4}\right)\right] \\
& -\frac{8}{9} g_{1}^{4}\left[8 \pi^{4} g_{1}^{6} \zeta(5)+21 \pi^{2}\left(11 g_{1}^{2}+5 g_{2}^{2}\right) g_{1}^{4} \zeta(7)+27\left(5 g_{1}^{6}(60 \zeta(3) \zeta(5)-91 \zeta(9))\right.\right. \\
& -g_{2}^{2} g_{1}^{4}(100 \zeta(3) \zeta(5)+371 \zeta(9))+g_{2}^{4} g_{1}^{2}(20 \zeta(3) \zeta(5)-161 \zeta(9)) \\
& \left.\left.\left.+g_{2}^{6}(100 \zeta(3) \zeta(5)-21 \zeta(9))\right)\right]\right\}+\mathcal{O}\left(g^{14}\right)
\end{aligned}
$$

for the term constant in $b-1$ and

$f_{1}^{(1)}\left(g_{1}^{2}, g_{2}^{2}\right)=-\left(g_{2}^{2}-g_{1}^{2}\right) \pi^{2}\left\{\frac{80}{3} g_{1}^{8} \zeta(5)+\frac{16}{9} g_{1}^{8}\left(16 \pi^{2} g_{1}^{2} \zeta(5)+21\left(11 g_{1}^{2}+5 g_{2}^{2}\right) \zeta(7)\right)\right\}+\mathcal{O}\left(g^{14}\right)$,

for the linear piece. In order to not overload the reader with information, we refrain from presenting any additional orders in the $(b-1)$ expansion, since they can be easily taken from (C.12).

A short remark is in order. The terms in the expansions (4.16) and (4.17) are homogeneous polynomials in the two couplings $g_{1}$ and $g_{2}$ of a given degree. By $\mathcal{O}\left(g^{n}\right)$, we mean that the results exclude polynomials of homogeneous degree greater or equal to $n$. Lastly, the expression for the other effective coupling $f_{2}\left(g_{1}^{2}, g_{2}^{2} ; b\right)$ can be obtained by using the $\mathbb{Z}_{2}$ cyclic symmetry of the theory

$$
f_{2}\left(g_{1}^{2}, g_{2}^{2} ; b\right)=f_{1}\left(g_{2}^{2}, g_{1}^{2} ; b\right)
$$

For the general $\mathbb{Z}_{r}$ cyclic quivers, we have the results

$$
\begin{aligned}
f_{k}^{(0)}\left(g_{i}^{2}\right)= & g_{k}^{2}+6 \zeta(3) g_{k}^{4}\left[g_{k-1}^{2}+g_{k+1}^{2}-2 g_{k}^{2}\right]-20 \zeta(5) g_{k}^{4}\left[g_{k-1}^{4}+g_{k+1}^{4}-6 g_{k}^{4}+2 g_{k}^{2}\left(g_{k-1}^{2}+g_{k+1}^{2}\right)\right] \\
& +g_{k}^{4}\left[70 \zeta(7)\left(g_{k-1}^{6}+g_{k+1}^{6}-16 g_{k}^{6}+3 g_{k}^{4}\left(g_{k-1}^{2}+g_{k+1}^{2}\right)+4 g_{k}^{2}\left(g_{k-1}^{4}+g_{k+1}^{4}\right)\right)\right. \\
& -2 \zeta(2)(20 \zeta(5)) g_{k}^{4}\left(g_{k-1}^{2}+g_{k+1}^{2}-2 g_{k}^{2}\right)+(6 \zeta(3))^{2}\left(8 g_{k}^{6}-2 g_{k-1}^{6}-2 g_{k+1}^{6}+g_{k-1}^{4} g_{k-2}^{2}\right. \\
& \left.\left.+g_{k+2}^{2} g_{k+1}^{4}-6 g_{k}^{4}\left(g_{k-1}^{2}+g_{k+1}^{2}\right)+2 g_{k}^{2}\left(g_{k-1}^{4}+g_{k-1}^{2} g_{k+1}^{2}+g_{k+1}^{4}\right)\right)\right]+\mathcal{O}\left(g^{12}\right)
\end{aligned}
$$


already present in $[2]$ and

$$
f_{k}^{(1)}\left(g_{i}^{2}\right)=80 g_{k}^{8}\left(2 g_{k}^{2}-g_{k+1}^{2}-g_{k-1}^{2}\right) \zeta(2) \zeta(5)+\mathcal{O}\left(g^{12}\right),
$$

for the first $b-1$ correction. The effective couplings for the general cyclic quivers are symmetric under $\mathbb{Z}_{r}$.

\subsection{Strong coupling results}

Similarly to the weak coupling approximation in subsection 4.1, we define the strong coupling regime to be the one in which all the couplings are large, i.e. we suppose that they all scale like (4.13) with $t \gg 1$.

In appendix D, we present the strong coupling analysis that we omitted in [2]. We obtain namely that, at the leading order, the densities $\rho_{k}$ behave like $\rho_{k}(x) \sim \frac{1}{2 \pi g_{k}^{2}} \sqrt{\mu_{k}^{2}-x^{2}}$ with the widths

$$
\mu_{k}=\bar{\mu}:=2 \sqrt{\frac{r g_{1}^{2} \cdots g_{r}^{2}}{\sum_{i=1}^{r} \prod_{k \neq i} g_{k}^{2}}} \quad \forall k .
$$

This implies that the Wilson loops expectation values asymptotically go like

$$
\left\langle W_{k}^{ \pm}\right\rangle \sim \frac{e^{2 \pi b^{ \pm 1} \bar{\mu}}}{2 \pi^{2} b^{ \pm 3 / 2} \bar{\mu}^{\frac{3}{2}}}+\mathcal{O}(b-1)^{2} .
$$

The leading piece in the couplings of the above can be written as

$$
\log \left\langle W_{k}^{ \pm}\right\rangle \sim 2 \pi \mu_{k} \pm(b-1)\left(2 \pi \mu_{k}-\frac{3}{2}\right)+O\left((b-1)^{2}\right)
$$

up to logarithmic corrections that are sub-leading and can be dropped. Due to the exponential term, the strong coupling limit of the effective couplings is simply given by comparing (D.31) with the width $\mu$ for $\mathcal{N}=4$. Since for $\mathcal{N}=4$ SYM we have $\mu^{2}=4 g^{2}$, comparing with (D.31) leads to

$$
f_{k}^{(0)}\left(g_{1}, \ldots, g_{r}\right)=r\left(\sum_{j=1}^{r} \frac{1}{g_{j}^{2}}\right)^{-1}=\frac{r g_{1}^{2} \cdots g_{r}^{2}}{\sum_{i=1}^{r} \prod_{k \neq i} g_{k}^{2}},
$$

up to constant and logarithmic corrections. Furthermore, to that order of precision $f_{k}^{(1)}$ is zero, which can be seen by plugging (4.23) into (4.14), solving for $f_{k}$.

\section{The Bremsstrahlung function and the entanglement entropy}

Having in section 4 derived the vacuum expectation value of the Wilson loops on the ellipsoid, it is now time to reap the fruits of our labor and investigate the quantities that we can easily obtain from them, namely the Bremsstrahlung function and the entanglement entropy. 


\subsection{The Bremsstrahlung function}

For $\mathcal{N}=4$, we can obtain the Wilson loop on the ellipsoid by simply making the substitution $g \rightarrow g b^{ \pm 1}$, leading to the planar limit result

$$
\left\langle W_{\mathcal{N}=4}^{ \pm}\left(g^{2} ; b\right)\right\rangle=\frac{I_{1}\left(4 \pi g b^{ \pm 1}\right)}{2 \pi g b^{ \pm 1}}+\mathcal{O}\left((b-1)^{2}\right),
$$

where $I_{n}$ are the modified Bessel functions of the first kind. It follows from (2.6) and (5.1) that we have in the planar limit the expression (see [21] for an earlier derivation of $B_{\mathcal{N}=4}$ )

$$
B_{\mathcal{N}=4}\left(g^{2}\right)=\frac{g\left(I_{0}(4 \pi g)+I_{2}(4 \pi g)\right)}{2 \pi I_{1}(4 \pi g)}-\frac{1}{4 \pi^{2}}=\frac{g I_{2}(4 g \pi)}{\pi I_{1}(4 g \pi)},
$$

where we have used $\frac{d}{d x} I_{1}=\frac{1}{2}\left(I_{0}+I_{2}\right)$. One can check that for large $g$ we have $B_{\mathcal{N}=4}(g) \sim$ $\frac{g}{\pi}-\frac{3}{8 \pi^{2}}$. In particular, $B_{\mathcal{N}=4}(g)$ is monotonically growing for all $g>0$ and is hence invertible in that domain. It follows that the equation $B_{\mathcal{N}=4}(x)=y$ has an unique solution for $y$ positive. We now define effective coupling $f_{B ; k}$ for the $\mathbb{Z}_{r}$ quiver theories by demanding

$$
B_{\mathcal{N}=4}\left(f_{B ; k}\left(g_{1}^{2}, \ldots, g_{r}^{2}\right)\right)=B_{k}\left(g_{1}^{2}, \ldots, g_{r}^{2}\right) .
$$

We find that, in the weak coupling $B_{k}$ goes like,

$$
\begin{aligned}
B_{k}= & g_{k}^{2}-\frac{2 \pi^{2}}{3} g_{k}^{4}+\frac{2}{3} g_{k}^{4}\left[\pi^{4} g_{k}^{2}+9 \zeta(3)\left(-2 g_{k}^{2}+g_{k+1}^{2}+g_{k-1}^{2}\right)\right] \\
& +\left[8 \pi^{2} \zeta(3)\left(2 g_{k}^{2}-g_{k+1}^{2}-g_{k-1}^{2}\right) g_{k}^{6}+20 \zeta(5)\left(6 g_{k}^{4}-2\left(g_{k+1}^{2}+g_{k-1}^{2}\right) g_{k}^{2}-g_{k+1}^{4}-g_{k-1}^{4}\right) g_{k}^{4}-\frac{32}{45} \pi^{6} g_{k}^{8}\right] \\
& +\left[12 \pi^{4}\left(-2 g_{k}^{2}+g_{k+1}^{2}+g_{k-1}^{2}\right) g_{k}^{8} \zeta(3)-\frac{40 \pi^{2}}{3}\left(10 g_{k}^{4}-3\left(g_{k+1}^{2}+g_{k-1}^{2}\right) g_{k}^{2}-2\left(g_{k+1}^{4}+g_{k-1}^{4}\right)\right) g_{k}^{6} \zeta(5)\right. \\
& +2 g_{k}^{4}\left(16 g_{k}^{6}\left(9 \zeta(3)^{2}-35 \zeta(7)\right)-3\left(g_{k+1}^{2}+g_{k-1}^{2}\right) g_{k}^{4}\left(36 \zeta(3)^{2}-35 \zeta(7)\right)\right. \\
& +4 g_{k}^{2}\left(g_{k+1}^{4}\left(9 \zeta(3)^{2}+35 \zeta(7)\right)+9 g_{k-1}^{2} g_{k+1}^{2} \zeta(3)^{2}+g_{k-1}^{4}\left(9 \zeta(3)^{2}+35 \zeta(7)\right)\right) \\
& \left.-36 g_{k-1}^{6} \zeta(3)^{2}+18 g_{k-2}^{2} g_{k-1}^{4} \zeta(3)^{2}+18 g_{k+1}^{4} g_{k+2}^{2} \zeta(3)^{2}+35 g_{k-1}^{6} \zeta(7)+g_{k+1}^{6}\left(35 \zeta(7)-36 \zeta(3)^{2}\right)\right) \\
& \left.+\frac{104}{135} \pi^{8} g_{k}^{10}\right]+\mathcal{O}\left(g^{12}\right) .
\end{aligned}
$$

For the $\mathbb{Z}_{2}$ quiver, the above can be checked from the explicit result (C.12) for the Wilson loop.

We now wish to discuss the relationships between all the different effective couplings. For the sake of clarity, we shall suppress the indices referring to the gauge groups. From the Wilson loops on the ellipsoids, we extract the effective coupling $f_{k}\left(g_{i}^{2} ; b\right)$ via (4.14). This defines the $b$-dependent effective coupling $f_{k}\left(g_{i}^{2} ; b\right)$. For $b=1$, it reduces to the "Wilson loop" effective couplings

$$
f_{W ; k}\left(g_{1}^{2}, \ldots, g_{r}^{2}\right):=f_{k}\left(g_{1}^{2}, \ldots, g_{r}^{2} ; b\right)_{\mid b=1} \text {. }
$$


that we used in [2]. On the other hand, from the Bremsstrahlung function, we can extract $f_{B ; k}\left(g_{i}^{2}\right)$ through (5.3). Let us see how the two are related. We have

$$
\begin{aligned}
B_{k}\left(g_{i}^{2}\right) & =\frac{1}{4 \pi^{2}} \frac{d}{d b} \log \left\langle W_{k}^{+}\left(g_{i}^{2} ; b\right)\right\rangle_{\mid b=1}=\frac{1}{4 \pi^{2}} \frac{d}{d b} \log \left\langle W_{\mathcal{N}=4}\left(f_{k}\left(g_{i}^{2} ; b\right) ; b\right)\right\rangle_{\mid b=1} \\
& =\left.\frac{1}{4 \pi^{2}} \frac{\partial}{\partial g^{2}} \log \left\langle W_{\mathcal{N}=4}\left(f_{W ; k}\left(g_{i}^{2}\right)\right)\right\rangle \frac{\partial f_{k}\left(g_{i}^{2} ; b\right)}{\partial b}\right|_{b=1}+B_{\mathcal{N}=4}\left(f_{W ; k}\left(g_{i}^{2}\right)\right)
\end{aligned}
$$

From [21], we take

$$
B_{\mathcal{N}=4}=\frac{\lambda}{2 \pi^{2}} \partial_{\lambda} \log \left\langle W_{\mathcal{N}=4}(\lambda)\right\rangle \Longrightarrow \frac{\partial}{\partial g^{2}} \log \left\langle W_{\mathcal{N}=4}\left(g^{2}\right)\right\rangle=\frac{2 \pi^{2}}{g^{2}} B_{\mathcal{N}=4}\left(g^{2}\right)
$$

Since $B_{\mathcal{N}=4}\left(f_{B ; k}\left(g_{i}^{2}\right)\right) \stackrel{!}{=} B_{k}\left(g_{i}^{2}\right)$, it follows that

$$
B_{\mathcal{N}=4}\left(f_{B ; k}\left(g_{i}^{2}\right)\right)=\left(1+\frac{f_{k}^{(1)}\left(g_{i}^{2}\right)}{2 f_{W ; k}\left(g_{i}^{2}\right)}\right) B_{\mathcal{N}=4}\left(f_{W ; k}\left(g_{i}^{2}\right)\right)
$$

where the first derivative $\partial_{b} f_{k}\left(g_{i}^{2} ; b\right)_{b=1} \equiv f_{k}^{(1)}\left(g_{i}^{2}\right)$ is given in (4.17) for the $\mathbb{Z}_{2}$ quiver and (4.20) in general. Hence, the discrepancy between $f_{B ; k}$ and $f_{W ; k}$ comes from the first derivative of the "full" effective coupling $f_{k}\left(g_{i}^{2} ; b\right)$ at $b=1$. To the order that we care to check, the discrepancies are always proportional to $\zeta(2 n)$.

In the strong coupling limit, the result (4.22) for the Wilson loop expectation values implies that the Bremsstrahlung function for the $k^{\text {th }}$ gauge group goes like

$$
B_{k} \sim \frac{\mu_{k}}{2 \pi}=\frac{1}{\pi} \sqrt{\frac{r g_{1}^{2} \cdots g_{r}^{2}}{\sum_{i=1}^{r} \prod_{k \neq i} g_{k}^{2}}}
$$

ignoring constant and logarithmic contributions, where the widths $\mu_{k}$ of the densities are to be found in (4.21). Since the leading contribution to $f_{k}^{(1)}$ is zero at strong coupling, $f_{B ; k}=f_{W ; k}$ to the precision we have in that regime.

\subsection{Entanglement entropy}

Combining the results of $[8,19]$, for $4 \mathrm{D} \mathcal{N}=2$ SCFTs, the additional entanglement entropy of a spherical region due to the presence of a heavy probe located at its origin is given by

$$
S=\log \langle W\rangle-8 \pi^{2} h_{W}=\left(1-\frac{2}{3} \partial_{b}\right)\left\langle\log W^{+}\right\rangle_{\mid b=1}=\log \langle W\rangle-\frac{8 \pi^{2}}{3} B .
$$

For $\mathcal{N}=4$, this result combined with expressions (5.1) and (5.2) gives

$$
S_{\mathcal{N}=4}\left(g^{2}\right)=\log \left(\frac{I_{1}(4 g \pi)}{2 \pi g}\right)-\frac{8 \pi g}{3} \frac{I_{2}(4 g \pi)}{I_{1}(4 g \pi)} .
$$

Unlike the Bremsstrahlung function $B_{\mathcal{N}=4}$ or the Wilson loop expectation $\log \left\langle W_{\mathcal{N}=4}\right\rangle$, the entanglement entropy $S_{\mathcal{N}=4}$ is not monotonically growing in $g$. Hence, we cannot for general values of the couplings find a single solution to the equation $S_{\mathcal{N}=4}\left(f_{k}\left(g_{i}^{2}\right)\right)=S_{k}\left(g_{i}^{2}\right)$, 
though of course we can do it in the weak or strong coupling limits. We restrict to simply stating the weak and strong coupling expansions of the entanglement entropies by using the results of the appendices $\mathrm{C}$ and $\mathrm{D}$. We find

$$
\begin{aligned}
S_{k}= & -\frac{2 \pi^{2}}{3} g_{k}^{2}+\frac{10}{9} \pi^{4} g_{k}^{4}-\frac{4}{3}\left[\pi^{2} g_{k}^{4}\left(3 \zeta(3)\left(-2 g_{k}^{2}+g_{k+1}^{2}+g_{k-1}^{2}\right)+\pi^{4} g_{k}^{2}\right)\right] \\
& +\frac{8 \pi^{2}}{135} g_{k}^{4}\left[-225 \pi^{2} \zeta(3)\left(2 g_{k}^{2}-g_{k+1}^{2}-g_{k-1}^{2}\right) g_{k}^{2}-225 \zeta(5)\left(6 g_{k}^{4}-2\left(g_{k+1}^{2}+g_{k-1}^{2}\right) g_{k}^{2}\right.\right. \\
& \left.\left.-g_{k+1}^{4}-g_{k-1}^{4}\right)+26 \pi^{6} g_{k}^{4}\right]+\mathcal{O}\left(g^{10}\right),
\end{aligned}
$$

in the weak coupling. Plugging (4.23) and (5.9) into (5.10), we get

$$
S_{k} \sim \frac{2 \pi}{3} \mu_{k}=\frac{4 \pi}{3} \sqrt{\frac{r g_{1}^{2} \cdots g_{r}^{2}}{\sum_{i=1}^{r} \prod_{k \neq i} g_{k}^{2}}} .
$$

up to constant and logarithmic contributions for the strong coupling limit, with $\mu_{k}$ taken from (4.21).

\section{Universality of the coupling substitution rule}

In this section we wish to argue that the effective couplings $f_{k}\left(g_{i}^{2}\right)$ that we have been calculating are universal, i.e. they are the same for any observable in the $\mathrm{SU}(2,1 \mid 2)$ sector, up to some scheme dependence that is related to the way the theory is regulated in the infrared. Firstly, it is important to recall that in $[1,2]$ we argued that in perturbation theory the functions $f_{k}\left(g_{i}^{2}\right)$ compute the finite renormalization of the $\mathcal{N}=2$ gluon propagator relative to the $\mathcal{N}=4$ one,

$$
f_{k}\left(g_{i}^{2}\right)=g_{k}^{2}+g_{k}^{2}\left[\left(\mathcal{Z}_{g_{k}}^{\mathcal{N}=2}\right)^{2}-\left(\mathcal{Z}_{g_{k}}^{\mathcal{N}=4}\right)^{2}\right] .
$$

We checked this proposal by a three loop calculation of the difference between the $\mathcal{N}=2$ and the $\mathcal{N}=4$ gluon propagator. The Feynman diagram result, originally done in [2], is presented in (2.9).

In sections 4 and 5 , we found that up to three loops ${ }^{11}$ in the weak coupling expansion and at leading order in the strong coupling the effective couplings are universal, i.e. the same for the different observables that we studied, namely the Wilson loop, the Bremsstrahlung function and the entanglement entropy. Hence, ${ }^{12}$ up to that order we see $f_{W}=f_{B}=f_{S}=$ $f$ with

$$
f_{1}\left(g_{i}^{2}\right)=\left\{\begin{array}{lr}
g_{1}^{2}+2\left(g_{2}^{2}-g_{1}^{2}\right)\left[6 \zeta(3) g_{1}^{4}+20 \zeta(5) g_{1}^{4}\left(g_{2}^{2}+3 g_{1}^{2}\right)+\cdots\right] & g_{1}, g_{2} \rightarrow 0 \\
2 \frac{g_{1}^{2} g_{2}^{2}}{g_{1}^{2}+g_{2}^{2}}+\cdots, & g_{1}, g_{2} \rightarrow \infty
\end{array},\right.
$$

\footnotetext{
${ }^{11}$ Three loops for $f\left(g_{i}^{2}\right)$ is four loops for the observables, the Wilson loop, the Bremsstrahlung function and the entanglement entropy. An insertion of a tree level propagator creates an one loop correction for them and so on.

${ }^{12}$ One can also derive an effective coupling $f_{S}$ for the entanglement entropy. Due to the definition (5.10), $f_{S}$ can be recovered from $f_{B}$ and $f_{W}$. Hence, it is enough to discuss the other effective couplings.
} 
for the $\mathbb{Z}_{2}$ quiver theory. These results are identical with the ones in [2] and thus in perturbation theory up to three loops the effective couplings are universal, and compute the relative finite renormalization of the gluon propagators (6.1). In the strong coupling the leading term matches the prediction of AdS/CFT (2.10), (2.11).

Starting at four loops, we found, see equation (5.8), that there are discrepancies between the different effective couplings for the different observables that are always proportional to $\zeta(2)=\frac{\pi^{2}}{6}$. The first few terms read

$\Delta f\left(g_{i}^{2}\right)=80 \zeta(2) g_{1}^{8}\left(g_{1}^{2}-g_{2}^{2}\right) \zeta(5)-g_{1}^{8}\left(g_{1}^{2}-g_{2}^{2}\right)\left(192 g_{1}^{2} \zeta(2)^{2} \zeta(5)+112\left(11 g_{1}^{2}+5 g_{2}^{2}\right) \zeta(2) \zeta(7)\right)+\cdots$.

Moreover, for the two observables $B$ and $\langle W\rangle$, the difference between the two effective couplings is due to the dependence in $b$,

$$
f_{B}-f_{W}=\Delta f \sim \frac{\partial f}{\partial b},
$$

see also equation (5.8). Beginning with this observation and stressing the fact that the parameter $b$ determines how we cut off the low energy momenta for a given calculation, as it is related to the size and shape of the ellipsoid, we wish to argue that the way we extract $f_{k}\left(g_{i}^{2}\right)$ suffers from scheme dependence originating in the way the theory is regulated in the infrared.

At the order $g^{10}$, where the discrepancies appear for the first time, the effective coupling reads

$$
\begin{aligned}
f_{W ; 1}\left(g_{1}, g_{2}\right)=\cdots & +2\left(g_{2}^{2}-g_{1}^{2}\right) g_{1}^{4}\left[70 \zeta(7)\left(g_{2}^{4}+5 g_{1}^{2} g_{2}^{2}+8 g_{1}^{4}\right)\right. \\
& \left.-40 \zeta(2) \zeta(5) g_{1}^{4}-2(6 \zeta(3))^{2}\left(g_{2}^{4}-g_{1}^{2} g_{2}^{2}+2 g_{1}^{4}\right)\right] .
\end{aligned}
$$

While it is very clear how to get from Feynman diagrams the $\zeta(7)$ and the $\zeta(3)^{2}$ pieces [2], it is not possible in flat space and for the massless and finite theories that we are considering to produce a $\zeta(2) \zeta(5)$ term by one or more Feynman diagrams. This can be understood by carefully looking at the classification of the massless four loop integrals [48]. The reader needs to keep in mind that the theories we are looking at are finite [49] and hence the poles always have to cancel. It would be very important to demonstrate this statement with an explicit calculation, but we leave this for future work.

Let us further stress that the way we have been computing the different $f_{k}\left(g_{i}^{2}\right)$ is through localization, which is always done on a sphere [4] or on an ellipsoid [14]. For those geometries, some of the fields couple conformally to the curvature acquiring an effective mass term $m^{2}=\mu_{R}^{2} \propto R \propto(\ell \tilde{\ell})^{-1}$, proportional to a scale set by the Ricci scalar $R$ of the ellipsoid. Hence, if we want to reproduce the $\zeta(2) \zeta(5)$ term by Feynman diagrams, we would have to take into account that some propagators in the integrals that we are computing become massive. These mass terms will then be renormalized. For generic theories, this conformal coupling to the curvature usually begins to renormalize starting at two loops. For theories with supersymmetry, the conformal coupling to the curvature will start to renormalize one loop later, at three loops, and we believe that in our case with 

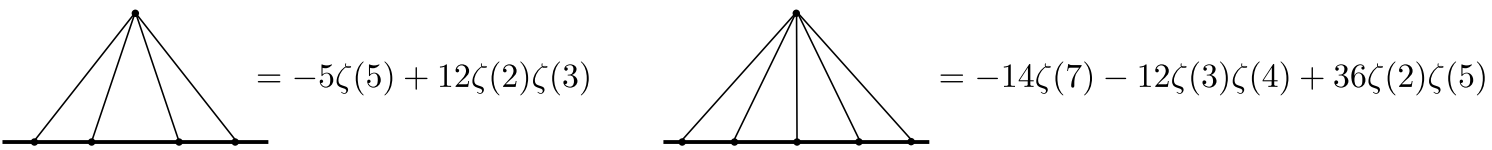

Figure 4. The $\zeta(2)$ s that we are looking for can be created by massive on-shell propagator diagrams. The thick lines indicate the massive propagators, while the thin lines stand for massless ones. The computation is done with the external massive particle momentum being on the mass shell.

$\mathcal{N}=2$ superconformal symmetry the effective mass term will start to renormalize at four loops. Moreover, the presence of massive modes in the loops forces us to specify a mass renormalization scheme. In the localization results, the size of the sphere or of the ellipsoid is a parameter independent of $g$. The mass term $m^{2}=\mu_{R}^{2} \propto R$ does not renormalize, but is instead kept fixed. This is a very particular scheme choice.

Our next step is to recall examples in 4D QFTs where terms proportional to $\zeta(2)$ are created. One instance that immediately comes to mind is the computation of bubbles with no external legs. A famous example is the Casimir effect, for which $\left\langle T_{00}\right\rangle=-\frac{\pi^{2}}{90 L^{4}}=$ $-\zeta(2) \frac{1}{15 L^{4}}$. A second common way to create $\zeta(2) \mathrm{s}$ is through mass renormalization, when the mass counterterm is inserted in a bigger diagram and in particular in the large mass expansion [50, 51]. Feynman diagrams like the ones depicted in figure 4 with some on shell propagators are known to create $\zeta(2) \zeta(3)$ or $\zeta(2) \zeta(5)$ terms. ${ }^{13}$ On the left hand side of figure 4, taken from [51], a $\zeta(2) \zeta(3)$ finite term is created. The $\zeta(2) \zeta(5)$ term that we are looking for is going to come from a diagram like the one depicted on the right side of figure 4 , at one loop higher than the $\zeta(2) \zeta(3)$ one.

Now that we have made a very particular choice for the mass renormalization scheme, we also have to UV regularize. Let's consider a UV cut off regularization with a UV scale $\Lambda_{\mathrm{UV}}$. From an effective field theory point of view, ${ }^{14}$ we should be able to perform the calculation either with $\Lambda_{\mathrm{UV}} \gg \mu_{R}$, or with $\Lambda_{\mathrm{UV}} \ll \mu_{R}$. In the latter case, we obtain an effective theory in which the massive fields have decoupled. The relation between the coupling constant in the effective theory without the heavy fields (EFT) and the full theory including the massive fields is given by the matching condition

$$
g_{\mathrm{EFT}}^{2}=z_{\text {match }} g_{\text {Full }}^{2},
$$

where $z_{\text {match }}$ is a function that depends on the choice of the scheme and the mass of the heavy fields. For us, the matching condition translates to a relation between the finite coupling renormalization factor $\mathcal{Z}_{g}^{\mathcal{N}=2}$ for the theory in the large mass expansion and the full theory

$$
\mathcal{Z}_{g \mathrm{EFT}}^{\mathcal{N}=2}=z_{\text {match }} \mathcal{Z}_{g \text { full }}^{\mathcal{N}=2} .
$$

See $[50,52]$ for the general ideology. Now in this theory with very massive fields, it is easy to see how to create the terms with $\zeta(2) \zeta(5)$. We just need to look again at the figure 4 . We

\footnotetext{
${ }^{13}$ We thank Erik Panzer for communicating to us the result of the integral on the right hand side of figure 4.

${ }^{14}$ We have in mind the well known relation between heavy quark QCD and the HQEF [52]. In particular, the decoupling theorem or the ramifications thereof, that is described in chapter 8 of [50].
} 
see that $\zeta(2) \zeta(3)$ terms can be created when we replace the propagator of the massive fields by the on shell one. It would be very beautiful to understand why there are no $\zeta(2) \zeta(3)$ terms at order $g^{8}$, or at any order that we have checked - it is most certainly a cancellation due to supersymmetry. The $\zeta(2) \zeta(5)$ term that we are looking for is expected to come from diagrams like the one depicted in the right of figure 4. These type of calculations can be done using [53] and we leave them for future work.

After all the arguments above, we come to the conclusion that the $\zeta(2)$ s stem from the fact that on the sphere or on the ellipsoid some of the fields have a mass term $m^{2}=\mu_{R}^{2} \propto R$. As we go up in loops, the mass is going to be renormalized forcing us to make a choice of a scheme. At the next loop order, this mass renormalization scheme choice is going to interfere with our $f\left(g_{i}\right)$ that computes the finite coupling renormalization of the coupling constant. This point was also addressed by Lewkowycz and Maldacena [8] and by Fraser in [54]. Specifically, in [8] it was discussed that the entanglement entropy computed has a finite ambiguity related to the precise procedure for defining the entropy with the additional finite contributions arising due to the conformal coupling of the scalars to the curvature.

Finally, we wish to conclude this section by connecting with our work a recent, independent result in favor of the existence of the universal coupling substitution rule. In $[55,56]$, the cusp anomalous dimension $\Gamma_{\text {cusp }}$ of QCD was compared with the one of $\mathcal{N}=4 \mathrm{SYM}$ and it was found that, upon replacing the coupling constant $g$ by the light-like cusp anomalous dimension $K$ as

$$
\Gamma_{\text {cusp }}\left(\varphi, g^{2}\right)=\Omega\left(\varphi, K\left(g^{2}\right)\right),
$$

the function $\Omega$ is independent of the choice of the theory, at least to three loops. All the dependence on the particular theory stands in $K$. It is very important to stress that this result is true for any $\varphi$. In (6.8), both $\Gamma_{\text {cusp }}$ and $K$ have to be computed in the same scheme. Expanding $\Omega$ in $\varphi$, we get

$$
\Omega\left(\varphi, K\left(g^{2}\right)\right)=\varphi^{2} \tilde{B}\left(K\left(g^{2}\right)\right)+\mathcal{O}\left(\varphi^{3}\right)
$$

and hence comparison with (2.5) leads to $B\left(g^{2}\right)=\tilde{B}\left(K\left(g^{2}\right)\right)$, where $\tilde{B}$ is an universal function, at least to 3 -loops. Since $B_{\mathcal{N}=4}\left(g^{2}\right)=\tilde{B}\left(K_{\mathcal{N}=4}\left(g^{2}\right)\right), B_{\mathcal{N}=2}\left(g^{2}\right)=\tilde{B}\left(K_{\mathcal{N}=2}\left(g^{2}\right)\right)$ and since from [1] we have the effective coupling relation $K_{\mathcal{N}=2}\left(g^{2}\right)=K_{\mathcal{N}=4}\left(f\left(g^{2}\right)\right)$, we can write

$$
B_{\mathcal{N}=2}\left(g^{2}\right)=\tilde{B}\left(K_{\mathcal{N}=4}\left(f\left(g^{2}\right)\right)=B_{\mathcal{N}=4}\left(f\left(g^{2}\right)\right) .\right.
$$

Following [55], this implies that, at least to 3-loops, the effective coupling $f\left(g^{2}\right)$ is universal, i.e. valid for $B$ as well as for the anomalous dimensions.

\section{Conclusions and outlook}

In this article, we calculated in the planar limit the vacuum expectation value of supersymmetric Wilson loops on ellipsoids for the $\mathcal{N}=2$ cyclic superconformal quivers, by using the localization formula of [14]. We provided explicit results, both in the weak and in the strong coupling limits. By comparing with $\mathcal{N}=4 \mathrm{SYM}$, we obtained the effective couplings $f_{W}\left(g_{i}^{2}\right)$ such that $W_{\mathcal{N}=4}\left(f_{W}\left(g_{i}^{2}\right)\right)=W_{\mathcal{N}=2}\left(g_{i}^{2}\right)$. Using (2.6) and (5.10) as an input, 
we extracted from the Wilson loops the Bremsstrahlung functions $B$ and the entanglement entropy $S$ and by comparing them with their $\mathcal{N}=4$ SYM counterparts we obtained the effective couplings $f_{B}\left(g_{i}^{2}\right)$ and $f_{S}\left(g_{i}^{2}\right)$.

From our calculations an important lesson emerges, namely that the effective couplings are universal, i.e. the same for the different observables, up to four loops in the weak coupling and for the leading order in the strong coupling. In particular, for the $\hat{A}_{1}$ or $\mathbb{Z}_{2}$ quiver theory, we find that the effective coupling of the first gauge group is

$$
f_{1}\left(g_{i}^{2}\right)=\left\{\begin{array}{lr}
g_{1}^{2}+2\left(g_{2}^{2}-g_{1}^{2}\right)\left[6 \zeta(3) g_{1}^{4}+20 \zeta(5) g_{1}^{4}\left(g_{2}^{2}+3 g_{1}^{2}\right)+\cdots\right] & g_{1}, g_{2} \rightarrow 0 \\
2 \frac{g_{1}^{2} g_{2}^{2}}{g_{1}^{2}+g_{2}^{2}}+\cdots, & g_{1}, g_{2} \rightarrow \infty
\end{array} .\right.
$$

This was checked against the Feynman diagrams calculation in the weak coupling [2], here reviewed in equations (2.8) and (2.9) as well as the strong coupling leading term (2.10) and (2.11). While this is not a direct check of the validity of (2.6) and (5.10), it shows that they are consistent with the coupling substitution rule.

Starting at five loops there are discrepancies (6.3) between the effective couplings for the different observables that are always proportional to $\zeta(2)$. The same exact observation was made by Fraser in [54] by comparing Wilson loops in different representations. In section 6 , we argued that the $\zeta(2)$ discrepancies are an artifact of the localization calculations being performed on the ellipsoid which imposes hard IR regulators and scheme dependence. Thus, we proposed that the effective couplings are universal up to the fact that one needs to properly take into account this scheme dependence. Following [1], any anomalous dimension in the purely gluonic, $\mathrm{SU}(2,1 \mid 2)$, sectors of $\mathcal{N}=2$ superconformal gauge theories can be obtained from the $\mathcal{N}=4$ results by directly replacing the $\mathcal{N}=4$ coupling by the effective couplings.

As an application of the substitution rule, we consider the calculation of the cusp anomalous dimension in $\mathcal{N}=2$ theories. For $\mathcal{N}=4 \mathrm{SYM}$, the light-like cusp anomalous dimension $K$ is given by

$$
K_{\mathcal{N}=4}\left(g^{2}\right)=4 g^{2}-\frac{4 \pi^{2} g^{4}}{3}+\frac{44 \pi^{4} g^{6}}{45}-\left(32 \zeta(3)^{2}+\frac{292 \pi^{6}}{315}\right) g^{8}+O\left(g^{10}\right)
$$

for small $g$ and $K_{\mathcal{N}=4}\left(g^{2}\right) \sim 2 g-\frac{3 \log (2)}{2 \pi}+\cdots$ for large $g$. Hence, inserting (4.19) and (4.24), we obtain the prediction for the $\mathbb{Z}_{2}$ quiver

$$
\begin{aligned}
K\left(g_{i}^{2}\right)= & 4 g_{1}^{2}-\frac{4 \pi^{2}}{3} g_{1}^{4}+\left[24 \zeta(3)\left(-2 g_{1}^{2}+g_{2}^{2}+g_{5}^{2}\right) g_{1}^{4}+\frac{44}{45} \pi^{4} g_{1}^{6}\right]-\left[32 \zeta(3)^{2} g_{1}^{8}\right. \\
& +16 \pi^{2} \zeta(3)\left(-2 g_{1}^{2}+g_{2}^{2}+g_{5}^{2}\right) g_{1}^{6}-80 \zeta(5)\left(6 g_{1}^{4}-2\left(g_{2}^{2}+g_{5}^{2}\right) g_{1}^{2}-g_{2}^{4}-g_{5}^{4}\right) g_{1}^{4} \\
& \left.+\frac{292}{315} \pi^{6} g_{1}^{8}\right]+\mathcal{O}\left(g^{10}\right)
\end{aligned}
$$

in the weak coupling regime and

$$
K\left(g_{i}^{2}\right) \sim 2 \sqrt{\frac{2 g_{1}^{2} g_{2}^{2}}{g_{1}^{2}+g_{2}^{2}}}
$$


in the strong coupling limit. Using the three-loop result of [55] and inserting $K$ into the function $\Omega$ in equation (6.8) provides us with the full $\Gamma_{\text {cusp }}(\varphi)$ function to three loops for $\mathcal{N}=2$ SCFTs.

There are many interesting questions and problems left for future work. In our mind, the number one priority is to perform an explicit Feynman diagram calculation on the sphere where the scalars acquire a mass and to explicitly find and compute the diagrams responsible for the first $\zeta(2) \zeta(5)$ discrepancies given in (6.3).

Wilson loops compute a big part of the data needed to obtain the high-energy scattering of charged particles $[57,58]$. Our diagrammatic studies lead us to believe that light-like polygonal Wilson loops stand a good change to obey the substitution rule, perhaps even in theories with less supersymmetry. Checking whether the substitution rule works by explicit Feynman diagrams calculations is an important direction worth pursuing. In investigating this direction, it will be paramount to use the appropriate superspace formalism. Moreover, in $\mathcal{N}=4 \mathrm{SYM}$, polygonal shaped Wilson loops are believed to be exactly dual to scattering amplitudes, see [59] and references therein for a recent review. This duality is due to the dual superconformal symmetry which is also believed to combine with the usual conformal symmetry to the Yangian of the full $\mathfrak{p s u}(2,2 \mid 4)$ integrable model. If the substitution rule works for light-like polygonal Wilson loops and gives the correct $\mathcal{N}=2$ results, it implies that there exists a Yangian symmetry acting on them, something worth checking. However, in strong contrast with $\mathcal{N}=4 \mathrm{SYM}$, the dual superconformal symmetry seems to break at two-loops ${ }^{15}[60,61]$ destroying the amplitude/Wilson loop duality. It would be very important to understand what this means for the integrability of the $\mathcal{N}=2$ SCFTs and to try to come up with ways to bypass this impasse.

In the case of $\mathcal{N}=4 \mathrm{SYM}$, the cusp anomalous dimension can be obtained by studying a supersymmetric Wilson loops with $L$ local fields inserted at the cusp [10,11]. This setup is described by TBA equations very similar to the ones of the spectral problem [10, 11]. However, these TBA equations are simpler and can be recast in terms of a matrix model [62-64] with a spectral curve that can be mapped to the classical string algebraic curve. For $\mathcal{N}=2$ SCFTs, it is currently not clear what happens beyond the $\mathrm{SU}(2,1 \mid 2)$ sector, but it is worth thinking whether it is possible to derive TBA equations for supersymmetric Wilson loops with local fields from the $\mathrm{SU}(2,1 \mid 2)$ sector inserted at the cusp.

So far, all the statements we have made about the substitution rule apply to operators long enough, i.e. with anomalous dimensions given by the asymptotic Bethe Ansatz, to evade wrapping corrections. From the TBA perspective, it is counterintuitive to expect that the substitution rule will remain valid when wrapping corrections are taken into account. Nevertheless, from the Feynman diagram point of view, it seems very plausible that the diagrammatic argument of [1] does hold in that case. It would be very illuminating to use Lüscher techniques [65] to investigate this further.

Another class of observables that is definitely worth studying is the correlation functions of chiral primary operators, studied in particular in [66-69]. The naive coupling sub-

\footnotetext{
${ }^{15}$ The dual superconformal symmetry is broken at two loops by terms depending non-trivially on the kinematics, which are suppressed in the Regge limit. Hence, the amplitude/Wilson loop duality that is broken at two-loops should be restored in the Regge limit.
} 
stitution for them does not work, due to the fact that finite terms from the non-holomorphic part of the $\mathcal{N}=2$ effective action $\int d^{8} \theta \mathcal{H}(\mathcal{W}, \overline{\mathcal{W}})$ contribute to the correlation functions. This is not the case for the anomalous dimensions in the $\mathrm{SU}(2,1 \mid 2)$ sector where effective vertices from $\int d^{8} \theta \mathcal{H}(\mathcal{W}, \overline{\mathcal{W}})$ cannot contribute [1]. Using the methods of [12, 13, 66-69], we can compute the exact Zamolodchikov metric, i.e. the metric in theory space, in the planar limit and from that recover the correlation functions of chiral primary operators. This is work in progress. The Zamolodchikov metric is another very interesting non-BPS observable. Investigating this direction is currently in progress.

While our results apply for the weak coupling of both the cyclic and the linear quivers, such as $\mathcal{N}=2$ SCQCD, see figure 1, they do not apply to the strong coupling of the linear quivers, since the limit $g_{r} \rightarrow 0$ does not commute with the strong coupling limit considered in section 4.2. As already discussed in [33], the strong coupling limit of $\mathcal{N}=2$ SCQCD is quite subtle. It would be important to understand more about the strong coupling limit of the linear quiver theories, in particular so as to improve our knowledge of their string duals.

Another theory in which a similar coupling substitution rule applies is ABJM, with the interpolating function $h(\lambda)$ also appearing in the magnon dispersion relation and being computed by comparing with localization techniques [70]. It would be very interesting to study the Kaluza-Klein reduction of $\mathcal{N}=4$ in the spirit of section 2 of [71], to see whether the interpolating function $h(\lambda)$ can be understood diagrammatically in a spirit similar to ours.

Last but not least, as we discussed in section 6 , in $[55,56]$ another, "experimental" coupling substitution rule was discovered, in which the coupling $g_{\mathrm{YM}}$ was replaced by the light-like cusp anomalous dimension $K$ and it was then found that, when so expressed, the full $\Gamma_{\text {cusp }}(\varphi)$ is independent of the specific particle content of the gauge theory, at least up to three loops. It would be very interesting to try to understand this fact using a diagrammatic argument of the form of [1], and to try to decide whether there should be other observables that could be obtained in similar ways.

Note added. As we have been finishing writing up this note, a closely related paper [72] appeared in the arXiv.

\section{Acknowledgments}

We thank James Drummond, Bartomeu Fiol, Johannes Henn, Zohar Komargodski, Sven Moch, Volker Schomerus, Matthias Steinhauser, Jörg Teschner, Konstantin Zarembo for useful discussions and feedback. We are grateful to Erik Panzer for sending us the integral on the right hand side of figure 4.

\section{A Chebyshev polynomials}

In this appendix, we summarize a couple of useful formulae involving the Chebyshev polynomials $T_{l}$ and $U_{l}$ of the first and second kind respectively. Important for us are the integral identities

$$
f_{-1}^{1} \sqrt{1-y^{2}} \frac{U_{k}(y)}{x-y} d y=\pi T_{k+1}(x), \quad f_{-1}^{1} \frac{1}{\sqrt{1-y^{2}}} \frac{T_{k}(y)}{x-y} d y=-\pi U_{k-1}(x) .
$$


which are only valid if $x \in(-1,1)$. The second part of equation (A.1) implies in particular for $k=1$

$$
f_{-\mu_{k}}^{\mu_{k}} \frac{d x}{\sqrt{\mu_{k}^{2}-x^{2}}} \frac{1}{z-x} x=-\pi .
$$

We can generalize (A.1) for arbitrary $x$ to to

$$
\begin{aligned}
& \frac{1}{\pi} f_{-\mu}^{\mu} \sqrt{\mu^{2}-y^{2}} \frac{U_{k}\left(\frac{y}{\mu}\right)}{x-y} d y=\mu T_{k+1}\left(\frac{x}{\mu}\right)-\operatorname{sgn}(x) \Theta(|x|-\mu) \sqrt{x^{2}-\mu^{2}} U_{k}\left(\frac{x}{\mu}\right), \\
& \frac{1}{\pi} f_{-\mu}^{\mu} \frac{1}{\sqrt{\mu^{2}-y^{2}}} \frac{T_{k}\left(\frac{y}{\mu}\right)}{x-y} d y=-\frac{1}{\mu} U_{k-1}\left(\frac{x}{\mu}\right)+\operatorname{sgn}(x) \Theta(|x|-\mu) \frac{T_{k}\left(\frac{x}{\mu}\right)}{\sqrt{x^{2}-\mu^{2}}},
\end{aligned}
$$

where $\Theta(x)=1$ if $x \geq 0$ and is zero otherwise is the Heaviside function. For $\mu>0$, one can prove for $x_{1} \neq x_{2}$

$$
\begin{aligned}
& \frac{1}{\pi} f_{-\mu}^{\mu} \frac{d y}{\sqrt{\mu^{2}-y^{2}}} \frac{1}{\left(x_{1}-y\right)\left(x_{2}-y\right)} \\
& \quad=\frac{\pi \delta\left(x_{1}-x_{2}\right) \Theta\left(\mu-\left|x_{1}\right|\right)}{\sqrt{\mu^{2}-x_{1}^{2}}}+\frac{1}{x_{1}-x_{2}}\left(\frac{\operatorname{sgn}\left(x_{2}\right) \Theta\left(\left|x_{2}\right|-\mu\right)}{\sqrt{x_{2}^{2}-\mu^{2}}}-\frac{\operatorname{sgn}\left(x_{1}\right) \Theta\left(\left|x_{1}\right|-\mu\right)}{\sqrt{x_{1}^{2}-\mu^{2}}}\right) .
\end{aligned}
$$

The identity (A.4) follows from (A.3) as well as from the observation: the equations (A.1) imply that for a function $\rho(x)$, that we can under some assumptions expand as $\rho(x)=$ $\sqrt{\mu^{2}-x^{2}} \sum_{n=0}^{\infty} c_{n} U_{n}\left(\frac{x}{\mu}\right)$, we can invert the finite Hilbert kernel and write a $\delta$-function relation like

$$
\rho(x)=-\frac{\sqrt{\mu^{2}-x^{2}}}{\pi^{2}} f_{-\mu}^{\mu} \frac{d y}{\sqrt{\mu^{2}-y^{2}}} \frac{1}{x-y} f_{-\mu}^{\mu} d z \frac{\rho(z)}{y-z} .
$$

\section{B Rewriting the partition functions}

We refer the reader to appendix $\mathrm{C}$ of [17] for a definition of the Barnes $\Gamma_{2}$ function, as well as the function $\Upsilon(x ; b)$. Here, we need the product formula for $\Gamma_{2}$. For that, we set for $\Re(s)>2$

$$
\chi\left(s ; \epsilon_{1}, \epsilon_{2}\right):=\sum_{n_{1}, n_{2} \geq 0}^{\prime} \frac{1}{\left(\epsilon_{1} n_{1}+\epsilon_{2} n_{2}\right)^{s}},
$$

where the prime removes the value $\left(n_{1}, n_{2}\right)=(0,0)$ from the sum. The function $\chi\left(s ; \epsilon_{1}, \epsilon_{2}\right)$ can be analytically continued for all $s \in \mathbb{C}$ except for $s=1$ and $s=2$ where there are poles. We have the residues

$$
\operatorname{Res}\left(\chi\left(s ; \epsilon_{1}, \epsilon_{2}\right), s=1\right)=\frac{1}{2}\left(\frac{1}{\epsilon_{1}}+\frac{1}{\epsilon_{2}}\right), \quad \operatorname{Res}\left(\chi\left(s ; \epsilon_{1}, \epsilon_{2}\right), s=2\right)=\frac{1}{\epsilon_{1} \epsilon_{2}}
$$


and the finite parts

$$
\begin{aligned}
\operatorname{Res}\left(\frac{\chi\left(s ; \epsilon_{1}, \epsilon_{2}\right)}{s-1}, s=1\right)= & -\frac{\log \epsilon_{1}}{\epsilon_{1}}+\frac{1}{2}\left(\frac{1}{\epsilon_{1}}-\frac{1}{\epsilon_{2}}\right) \log \epsilon_{2}+\frac{\gamma}{\epsilon_{1}}+\frac{\gamma}{2 \epsilon_{2}}-\frac{1}{2 \epsilon_{1}} \log 2 \pi \\
& -\frac{i}{b} \int_{0}^{\infty} \frac{\psi\left(i \frac{\epsilon_{1}}{\epsilon_{2}} y+1\right)-\psi\left(-i \frac{\epsilon_{1}}{\epsilon_{2}} y+1\right)}{e^{2 \pi y}-1} d y \\
\operatorname{Res}\left(\frac{\chi\left(s ; \epsilon_{1}, \epsilon_{2}\right)}{s-2}, s=2\right)= & \frac{\zeta(2)}{\epsilon_{1}^{2}}+\frac{\zeta(2)}{2 \epsilon_{2}^{2}}+\frac{1}{\epsilon_{1} \epsilon_{2}}\left(\gamma-1-\log \epsilon_{2}\right) \\
& -\frac{i}{\epsilon_{2}} \int_{0}^{\infty} \frac{\zeta_{H}\left(2, i \frac{\epsilon_{1}}{\epsilon_{2}} y+1\right)-\zeta_{H}\left(2,-i \frac{\epsilon_{1}}{\epsilon_{2}} y+1\right)}{e^{2 \pi y}-1} d y
\end{aligned}
$$

where $\psi$ is the digamma function, $\gamma$ is the Euler - Mascheroni constant and $\zeta_{H}(s, q)$ is the Hurwitz- $\zeta$ function with $(\Re(s)>1$ and $\Re(q)>0) \zeta_{H}(s, q):=\sum_{n=0}^{\infty} \frac{1}{(q+n)^{s}}$. Finally, using the shorthands

$r_{\chi}:=\operatorname{Res}\left(\frac{\chi\left(s ; \epsilon_{1}, \epsilon_{2}\right)}{s-1}, s=1\right), \quad s_{\chi}:=\operatorname{Res}\left(\frac{\chi\left(s ; \epsilon_{1}, \epsilon_{2}\right)}{s-2}, s=2\right)+\operatorname{Res}\left(\chi\left(s ; \epsilon_{1}, \epsilon_{2}\right), s=2\right)$,

we present the product formula for $\Gamma_{2}$

$$
\Gamma_{2}\left(x ; \epsilon_{1}, \epsilon_{2}\right)=\frac{e^{-r_{\chi} x+\frac{s_{\chi} x^{2}}{2}}}{x} \prod_{n_{1}, n_{2} \geq 0}^{\prime} \frac{e^{\frac{x}{\epsilon_{1} n_{1}+\epsilon_{2} n_{2}}-\frac{x^{2}}{2\left(\epsilon_{1} n_{1}+\epsilon_{2} n_{2}\right)^{2}}}}{1+\frac{x}{\epsilon_{1} n_{1}+\epsilon_{2} n_{2}}} .
$$

Let us introduce two new special functions that we need for (3.8). Specifically, we define

$$
\begin{aligned}
H_{v}(x ; b):= & \prod_{m, n=0}^{\infty} \sqrt{\left(1+\frac{x^{2}}{\left(b(m+1)+b^{-1} n\right)^{2}}\right)\left(1+\frac{x^{2}}{\left(b m+b^{-1}(n+1)\right)^{2}}\right)} \\
& \times e^{-\frac{x^{2}}{2\left(b(m+1)+b^{-1} n\right)^{2}}-\frac{x^{2}}{2\left(b m+b^{-1}(n+1)\right)^{2}}}, \\
H_{h}(x ; b):= & e^{-\zeta(2) \frac{b^{2}+b^{-2}}{2} x^{2}} \prod_{m, n=0}^{\infty}\left(1+\frac{x^{2}}{\left(b(m+1 / 2)+b^{-1}(n+1 / 2)\right)^{2}}\right) e^{-\frac{x^{2}}{\left(b(m+1)+b^{-1}(n+1)\right)^{2}}} .
\end{aligned}
$$

It is easy to see that both these functions are even in $x$, that $H_{v}(0 ; b)=H_{h}(0 ; b)=1$ and that

$$
\lim _{b \rightarrow 1} H_{v}(x ; b)=\lim _{b \rightarrow 1} H_{h}(x ; b)=H(x)=\prod_{n=1}^{n}\left(1+\frac{x^{2}}{n^{2}}\right)^{n} e^{-\frac{x^{2}}{n}} .
$$

Furthermore, $H_{a}(x ; b)=H_{a}\left(x ; b^{-1}\right)$, both for $a=v$ and for $a=h$. We also need to consider the logarithms of the functions $H_{v}$ and $H_{h}$ defined in (B.6). Let us define

$$
K_{v}(x ; b):=-\frac{d}{d x} \log \left(H_{v}(x ; b)\right), \quad K_{h}(x ; b):=-\log \frac{d}{d x} \log \left(H_{h}(x ; b)\right) .
$$

We can easily compute the logarithms of $H_{v}$ and $H_{h}$, finding that

$$
\begin{aligned}
& K_{v}(x ; b)=-2 \sum_{n=1}^{\infty}(-1)^{n} x^{2 n+1} \frac{\zeta_{2}\left(b, 2 n+2 ; b, b^{-1}\right)+\zeta_{2}\left(b^{-1}, 2 n+2 ; b, b^{-1}\right)}{2}, \\
& K_{h}(x ; b)=-2 \sum_{n=1}^{\infty}(-1)^{n} x^{2 n+1} \zeta_{2}\left(\left(b+b^{-1}\right) / 2,2 n+2 ; b, b^{-1}\right),
\end{aligned}
$$


where we have used Barnes $\zeta_{2}$ function $\zeta_{2}\left(x, s ; \epsilon_{1}, \epsilon_{2}\right):=\sum_{n_{1}, n_{2} \geq 0} \frac{1}{\left(x+n_{1} \epsilon_{1}+n_{2} \epsilon_{2}\right)^{s}}$, convergent for $\operatorname{Re}(s) \geq 2$. We need the following special values

$$
\begin{aligned}
\zeta_{2}(1,2 n+2 ; 1,1) & =\zeta(2 n+1), \\
\left.\frac{d}{d b} \zeta_{2}\left(b, 2 n+2 ; b, b^{-1}\right)\right|_{b=1} & =-(2 n+2) \zeta(2 n+2), \\
\left.\frac{d}{d b} \zeta_{2}\left(b^{-1}, 2 n+2 ; b, b^{-1}\right)\right|_{b=1} & =(2 n+2) \zeta(2 n+2), \\
\frac{d}{d b} \zeta_{2}\left(\left(b+b^{-1}\right) / 2,2 n+2 ; b, b^{-1}\right)_{\left.\right|_{b=1}} & =0 .
\end{aligned}
$$

Hence $K_{v}(x ; 1)=K_{h}(x ; 1)=K(x):=-2 \sum_{n=1}^{\infty}(-1)^{n} \zeta(2 n+1) x^{2 n+1}$ and

$$
\frac{d}{d b} K_{v}(x ; b)_{\mid b=1}=\frac{d}{d b} K_{h}(x ; b)_{\mid b=1}=0 .
$$

Similarly, expanding in $b$ around $b=1$, replacing the summation variables as $n_{1}=(r+s) / 2$, $n_{2}=(r-s) / 2$ with $r \in \mathbb{N}_{0}$ and $s \in\{-r,-r+2, \ldots, r\}$, we find the expansions

$$
\begin{aligned}
K_{v}(x ; b)= & -2 \sum_{n=1}^{\infty}(-1)^{n} x^{2 n+1}\left[\zeta(2 n+1)+\frac{4}{3}(n+1)(n \zeta(2 n+1)+(2 n+3) \zeta(2 n+3))(b-1)^{2}\right. \\
& \left.-4(n+1)(n \zeta(2 n+1)+(2 n+3) \zeta(2 n+3))(b-1)^{3}+\mathcal{O}(b-1)^{4}\right], \\
K_{h}(x ; b)= & -2 \sum_{n=1}^{\infty}(-1)^{n} x^{2 n+1}\left[\zeta(2 n+1)+\frac{2}{3}(n+1)(2 n \zeta(2 n+1)-(2 n+3) \zeta(2 n+3))(b-1)^{2}\right. \\
& \left.-2(n+1)(2 n \zeta(2 n+1)-(2 n+3) \zeta(2 n+3))(b-1)^{3}+\mathcal{O}(b-1)^{4}\right],
\end{aligned}
$$

where we have used $\left(B_{k}(1)\right.$ is the value of the $k^{\text {th }}$ Bernoulli polynomial at 1$)$

$$
\sum_{\substack{s=-r \\
\text { step 2 }}}^{r} s^{m}=\delta_{(m \bmod 2), 0}\left(\delta_{m, 0}+\frac{2^{m+1}}{m+1} \sum_{k=0}^{m} B_{k}(1)\left(\begin{array}{c}
m+1 \\
k
\end{array}\right)\left(\frac{r}{2}\right)^{m+1-k}\right) .
$$

Let us now return to the partition functions on the ellipsoid. For conformal field theories, we can claim that we can rewrite (3.4) as (3.7) with $Z_{1 \text {-loop }}^{\text {vect }}$ and $Z_{1 \text {-loop }}^{\text {hyper }}$ given in (3.8).

Proof. We rescale the integration variables in (3.4) by $\sqrt{\ell \tilde{\ell}}$. This does not change the results for conformal field theories. We now consider the partition functions (3.6) separately. First, we look at the vector multiplet contributions. We use the definitions of appendix $\mathrm{C}$ of [17] to write

$$
\Upsilon(x ; b)=-x b^{\left(b-b^{-1}\right) x} \frac{\Gamma(-b x) \Gamma\left(-b^{-1} x\right) \Gamma_{2}\left(Q / 2 ; b, b^{-1}\right)^{2}}{2 \pi \Gamma_{2}\left(x ; b, b^{-1}\right) \Gamma_{2}\left(-x ; b, b^{-1}\right)},
$$

in order to split away the Vandermonde determinant contribution. We get

$$
\begin{aligned}
Z_{1 \text { loop }}^{\text {vect }} & =\prod_{i<j=1}^{N} \Upsilon\left(i\left(a_{i}-a_{j}\right) ; b\right) \Upsilon\left(-i\left(a_{i}-a_{j}\right) ; b\right) \\
& =\prod_{i<j=1}^{N}\left(a_{i}-a_{j}\right)^{2} \frac{\prod_{s= \pm}\left[\Gamma\left(s i b\left(a_{i}-a_{j}\right)\right) \Gamma\left(s i b^{-1}\left(a_{i}-a_{j}\right)\right)\right] \Gamma_{2}(Q / 2)^{4}}{(2 \pi)^{2} \Gamma_{2}\left(i\left(a_{i}-a_{j}\right)\right)^{2} \Gamma_{2}\left(-i\left(a_{i}-a_{j}\right)\right)^{2}}
\end{aligned}
$$


Since we are only interested in the computation of the Wilson loops (3.5), we should be able to rescale the partition function even by an $b$-dependent function, so that we can drop the $\Gamma_{2}(Q / 2)^{4} /(2 \pi)^{2}$ part. Hence, we can use instead

$$
Z_{1-\text { loop }}^{\text {vect }}=\prod_{i<j=1}^{N}\left(a_{i}-a_{j}\right)^{2} \prod_{i<j=1}^{N} \frac{\Gamma\left(i b\left(a_{i}-a_{j}\right)\right) \Gamma\left(-i b\left(a_{i}-a_{j}\right)\right)}{\Gamma_{2}\left(i\left(a_{i}-a_{j}\right)\right) \Gamma_{2}\left(-i\left(a_{i}-a_{j}\right)\right)} \prod_{i<j=1}^{N}\left(b \leftrightarrow b^{-1}\right)
$$

Using (B.5) and $\Gamma(x)=\frac{e^{-\gamma x}}{x} \prod_{n=1}^{\infty} \frac{e^{\frac{x}{n}}}{1+\frac{x}{n}}$, we find

$$
\frac{\Gamma(i b x) \Gamma(-i b x)}{\Gamma_{2}(i x) \Gamma_{2}(-i x)}=\frac{e^{s_{\chi} x^{2}}}{b^{2}} \frac{\prod_{n=1}^{\infty}\left(1+\frac{x^{2}}{\left(b^{-1} n\right)^{2}}\right)}{\prod_{m, n \geq 0}^{\prime} \frac{e^{\frac{x^{2}}{\left(b m+b^{-1} n\right)^{2}}}}{1+\frac{x^{2}}{\left(b m+b^{-1} n\right)^{2}}}},
$$

where $s_{\chi}$ was defined in (B.4). Hence it follows that we can write (B.15) as

$$
Z_{1 \text {-loop }}^{\text {vect }}=\prod_{i<j=1}^{N}\left(a_{i}-a_{j}\right)^{2} \prod_{i, j=1}^{N} \prod_{i<j=1}^{N} e^{\left(s_{\chi}-\frac{b^{2}+b^{-2}}{2} \zeta(2)\right)\left(a_{i}-a_{j}\right)^{2}} H_{v}\left(a_{i}-a_{j} ; b\right) .
$$

Second, let us look at the hyper multiplet contribution. We write down explicitly

$$
\Upsilon(i x+Q / 2)=\frac{\Gamma_{2}(Q / 2)^{2}}{\Gamma_{2}(Q / 2+i x) \Gamma_{2}(Q / 2-i x)}
$$

and use (B.5) to get

$$
\begin{aligned}
\Upsilon(i x+Q / 2) & =e^{x^{2} s_{\chi}} \frac{(Q / 2+i x)(Q / 2-i x)}{(Q / 2)^{2}} \prod_{m, n \geq 0}^{\prime} e^{-\frac{x^{2}}{\left(b m+b^{-1} n\right)^{2}}} \frac{\left(1+\frac{Q / 2+i x}{b m+b^{-1} n}\right)\left(1+\frac{Q / 2-i x}{b m+b^{-1} n}\right)}{\left(1+\frac{Q / 2}{b m+b^{-1} n}\right)^{2}} \\
& =e^{x^{2} s_{\chi}}\left(1+\frac{4 x^{2}}{Q^{2}}\right) \prod_{m, n \geq 0}^{\prime} e^{-\frac{x^{2}}{\left(b m+b^{-1} n\right)^{2}}}\left(1+\frac{x^{2}}{\left(b(m+1 / 2)+b^{-1}(n+1 / 2)\right)^{2}}\right) .
\end{aligned}
$$

At this point, we can split the product for the exponential pieces as

$$
\prod_{m, n=0}^{\infty}(\cdots)=\prod_{m, n=1}^{\infty}(\cdots) \prod_{m=1}^{\infty}(\cdots) \prod_{n=1}^{\infty}(\cdots)
$$

express the $\prod_{m=1}^{\infty}(\cdots) \prod_{n=1}^{\infty}(\cdots)$ piece as $e^{-x^{2}\left(b^{2}+b^{-2)} \zeta(2)\right.}$ and absorb the factor $\left(1+\frac{4 x^{2}}{Q^{2}}\right)$ inside the remaining product to obtain

$$
\begin{aligned}
\Upsilon(i x+Q / 2) & =e^{x^{2}\left(2 s_{\chi}-\left(b^{2}+b^{-2}\right) \zeta(2)\right)} \prod_{m, n=0}^{\infty}\left(1+\frac{x^{2}}{\left(b(m+1 / 2)+b^{-1}(n+1 / 2)\right)^{2}}\right) e^{-\frac{x^{2}}{\left(b(m+1)+b^{-1}(n+1)\right)^{2}}} \\
& =e^{x^{2}\left(s_{\chi}-\frac{b^{2}+b^{-2}}{2} \zeta(2)\right)} H_{h}(x ; b) .
\end{aligned}
$$


Hence, feeding (B.21) into (3.6) leads to

$$
Z_{1 \text {-loop }}^{\text {hyper }}=\prod_{i, j=1}^{N} \Upsilon\left(i\left(a_{i}^{(1)}-a_{j}^{(2)}\right)+Q / 2 ; b\right)^{-1}=\prod_{i, j=1}^{N} e^{-\left(a_{i}^{(1)}-a_{j}^{(2)}\right)^{2}\left(s_{\chi}-\frac{b^{2}+b^{-2}}{2}\right)} H_{h}\left(a_{i}^{(1)}-a_{j}^{(2)} ; b\right)^{-1} .
$$

Putting (B.17) and (B.22) together, the exponential terms cancel for conformal field theories, leaving us with the desired result (3.8).

\section{The weak coupling expansion}

In this appendix, we wish to take the set of linear integral equations (4.10) and find an approximate solution for small values of the couplings. Our computations follow the principles outlined in [43]. For our purposes, we fix an integer $P \geq 1$ and expand the kernels $K_{v}$ and $K_{h}$ as

$$
K_{a}(x) \approx-2 \sum_{n=1}^{P}(-1)^{n} \mathrm{k}_{a}(n) x^{2 n+1},
$$

where $a \in\{v, h\}$, the coefficients $\mathrm{k}_{a}(n)$ can be extracted from equation (B.8) and we have suppressed the $b$ dependence. This expansion is sufficient in order to obtain the results up to order $g^{2(P+1)}$ in the couplings. We have for a given eigenvalue density $\rho_{k}$ the expression

$$
\int_{-\mu}^{\mu} d z \rho_{k}(z) K_{a}(y-z)=-2 \sum_{n=1}^{P}(-1)^{n} \mathbf{k}_{a}(n) \sum_{s=0}^{n}\left(\begin{array}{c}
2 n+1 \\
2 s
\end{array}\right) y^{2(n-s)+1} \mathfrak{m}_{2 s}^{(k)},
$$

where $\mathfrak{m}_{i}^{(k)}$ is the $i$-th moment of the density $\rho_{k}$, i.e.

$$
\int_{-\mu}^{\mu} \rho_{k}(x) x^{i}=\mathfrak{m}_{i}^{(k)} .
$$

Observe that the odd moments have to vanish due to the symmetry of the densities. Using Chebyshev polynomials, we can derive the integral formula

$$
f_{-\mu}^{\mu} \frac{d y}{x-y} \frac{y^{n}}{\sqrt{\mu^{2}-y^{2}}}=-\pi \sum_{t=0}^{\left\lfloor\frac{n-1}{2}\right\rfloor} \frac{(t+1) C_{t}}{4^{t}} \mu^{2 r} x^{n-1-2 t} .
$$

where $C_{r}=\frac{1}{r+1}\left(\begin{array}{c}2 r \\ r\end{array}\right)$ is the $r$-th Catalan number. Plugging (C.2) and (C.4) into (4.10), we the following result for the $k^{\text {th }}$ density:

$$
\begin{aligned}
\rho_{k}(x)= & \frac{1}{2 \pi g_{k}^{2}} \sqrt{\mu_{k}^{2}-x^{2}}-\frac{2}{\pi} \sqrt{\mu_{k}^{2}-x^{2}} \sum_{n=1}^{P}(-1)^{n} \sum_{l=1}^{r}\left[\delta_{k l} \mathrm{k}_{v}(n)-\frac{\delta_{k, l+1}+\delta_{k, l-1}}{2} \mathrm{k}_{h}(n)\right] \\
& \times \sum_{j=0}^{n}\left(\begin{array}{c}
2 n+1 \\
2 j
\end{array}\right) \mathfrak{m}_{2 j}^{(l)} \sum_{t=0}^{n-j} \frac{(t+1) C_{t}}{4^{t}} \mu_{k}^{2 t} x^{2(n-j-t)} .
\end{aligned}
$$


We thus have expressed each of the densities $\rho_{k}$ as functions of its $P$ first non-trivial moments $\mathfrak{m}_{2 i}^{(k)}, i=1, \ldots, P$. Computing the moments by plugging (C.5) into the definition (C.3), we obtain a set of $r \times P$ linear equations for the same number of variables $\mathfrak{m}_{2 i}^{(k)}$ :

$$
\begin{gathered}
\sum_{j=1}^{P} \sum_{l=1}^{r}\left[\delta_{i j} \delta_{k l}+\sum_{n=j}^{P}(-1)^{n}\left[\delta_{k l} \mathrm{k}_{v}(n)-\frac{\delta_{k, l+1}+\delta_{k, l-1}}{2} \mathrm{k}_{h}(n)\right]\left(\begin{array}{c}
2 n+1 \\
2 j
\end{array}\right)\right. \\
\left.\times \frac{\mu_{k}^{2(n+i-j+1)}}{4^{n+i-j}}\left(\begin{array}{c}
2 i \\
i
\end{array}\right)\left(\begin{array}{c}
2(n-j) \\
n-j
\end{array}\right) \frac{2(n-j)+1}{n-j+i+1}\right] \mathfrak{m}_{2 j}^{(l)} \\
=\frac{1}{g_{k}^{2}} \frac{C_{i}}{4^{i+1}} \mu_{k}^{2(i+1)}-\sum_{n=1}^{P}(-1)^{n}\left[\mathrm{k}_{v}(n)-\mathrm{k}_{h}(n)\right] \frac{\mu_{k}^{2(n+i+1)}}{4^{n+i}}\left(\begin{array}{c}
2 i \\
i
\end{array}\right)\left(\begin{array}{c}
2 n \\
n
\end{array}\right) \frac{2 n+1}{n+i+1},
\end{gathered}
$$

for $i=1, \ldots, P$ and $k=1, \ldots, r$. In (C.6), we have used the integral formula

$$
\int_{-\mu}^{\mu} d x \sqrt{\mu^{2}-x^{2}} x^{2 s}=\frac{2 \pi C_{s}}{4^{s+1}} \mu^{2(s+1)}
$$

the fact that $m_{0}^{(l)}=1 \forall l$ and the following formula for the Catalan numbers

$$
\sum_{t=0}^{m}(t+1) C_{t} C_{m+i-t}=\left(\begin{array}{c}
2 i \\
i
\end{array}\right)\left(\begin{array}{c}
2 m \\
m
\end{array}\right) \frac{2 m+1}{m+i+1} .
$$

Observe that the last line of (C.6) vanishes for $b=1$, since in that case $\mathrm{k}_{v}(n)=\mathrm{k}_{h}(n)$. The set of linear equations (C.6) allows us to solve for the moments as functions of the densities widths $\mu_{k}$ and of the couplings $g_{k}^{2}$. The $\mu_{k}$ are then expressed as functions of the coupling by normalizing the densities. Specifically, plugging (C.5) into the normalization condition for the densities (4.8), we arrive at

$$
\begin{aligned}
1= & \frac{\mu_{k}^{2}}{4 g_{k}^{2}}-\sum_{l=1}^{r} \sum_{n=1}^{P}(-1)^{n}\left[\delta_{k l} \mathrm{k}_{v}(n)-\frac{\delta_{k, l+1}+\delta_{k, l-1}}{2} \mathrm{k}_{h}(n)\right] \\
& \times \sum_{j=1}^{n}\left(\begin{array}{c}
2 n+1 \\
2 j
\end{array}\right) \frac{\mu_{k}^{2(n-j+1)} \mathfrak{m}_{2 j}^{(l)}}{4^{n-j}}\left(\begin{array}{c}
2(n-j) \\
n-j
\end{array}\right) \frac{2(n-j)+1}{n-j+1} \\
& -\sum_{n=1}^{P}(-1)^{n}\left[\mathrm{k}_{v}(n)-\mathrm{k}_{h}(n)\right] \frac{\mu_{k}^{2(n+1)}}{4^{n}}\left(\begin{array}{c}
2 n \\
n
\end{array}\right) \frac{2 n+1}{n+1} .
\end{aligned}
$$

Again, the last term of (C.9) vanishes for $b=1$. Inserting in (C.9) the expressions obtained from (C.6) for the moments allows us to solve for the $\mu_{k}$ as functions of the $g_{k}^{2}$.

In order to solve equations (C.6) and (C.9), it is numerically good to linearize in the couplings as

$$
\mu_{k}=2 g_{k}\left(1+\sum_{i=1}^{P+1} \alpha_{i}^{(k)} g_{k}^{2 i}\right), \quad \mathfrak{m}_{2 i}^{(k)}=C_{i} g_{k}^{2 i}\left(1+\sum_{j=1}^{P+1-i} \beta_{j, i}^{(k)} g_{k}^{2 i}\right) .
$$

We then expand the equations in powers of $g_{k}$ up to the power $2(P+1)$. Once we have solved for the coefficients $\alpha_{i}^{(k)}$ and the $\beta_{j, i}^{(k)}$, we have obtained the widths and the moments 
and the Wilson loop expectation values (4.12) can be expressed as

$$
\left\langle W_{k}^{ \pm}\right\rangle=\sum_{n=0}^{\infty} \frac{(2 \pi)^{2 n}}{(2 n) !} b^{ \pm 2 n} \mathfrak{m}_{2 n}^{(k)}=2 \sum_{n=0}^{\infty} \frac{(-1)^{n+1} \zeta(2 n)}{B_{2 n}} b^{ \pm 2 n} \mathfrak{m}_{2 n}^{(k)},
$$

where $B_{n}$ are the Bernoulli numbers.

For the $\mathbb{Z}_{2}$ quiver, we obtain the expansion up to order $(b-1)^{2}$

$$
\begin{aligned}
\left\langle W_{1}^{+}\right\rangle= & +2 \pi^{2} g_{1}^{2}+\frac{4}{3} \pi^{4} g_{1}^{4}+\frac{4}{9} \pi^{2} g_{1}^{4}\left[54 \zeta(3)\left(g_{2}^{2}-g_{1}^{2}\right)+\pi^{4} g_{1}^{2}\right] \\
& +\frac{4}{45} \pi^{2} g_{1}^{4}\left[360 \pi^{2} \zeta(3)\left(g_{2}^{2}-g_{1}^{2}\right) g_{1}^{2}+900 \zeta(5)\left(g_{1}-g_{2}\right)\left(g_{1}+g_{2}\right)\left(3 g_{1}^{2}+g_{2}^{2}\right)+\pi^{6} g_{1}^{4}\right] \\
& +\frac{8}{675} \pi^{2} g_{1}^{4}\left[-1350 \pi^{4} \zeta(3)\left(g_{1}^{2}-g_{2}^{2}\right) g_{1}^{4}+2250 \pi^{2} \zeta(5)\left(13 g_{1}^{4}-9 g_{2}^{2} g_{1}^{2}-4 g_{2}^{4}\right) g_{1}^{2}\right. \\
& \left.+675\left(g_{1}^{2}-g_{2}^{2}\right)\left(36 \zeta(3)^{2}\left(2 g_{1}^{4}-g_{2}^{2} g_{1}^{2}+g_{2}^{4}\right)-35 \zeta(7)\left(8 g_{1}^{4}+5 g_{2}^{2} g_{1}^{2}+g_{2}^{4}\right)\right)+\pi^{8} g_{1}^{6}\right]+\cdots \\
& +\left[4 \pi^{2} g_{1}^{2}+\frac{16}{3} \pi^{4} g_{1}^{4}+\frac{8}{3} \pi^{2} g_{1}^{4}\left[18 \zeta(3)\left(g_{2}^{2}-g_{1}^{2}\right)+\pi^{4} g_{1}^{2}\right]\right. \\
& +\frac{32}{45} \pi^{2} g_{1}^{4}\left[180 \pi^{2} \zeta(3)\left(g_{2}^{2}-g_{1}^{2}\right) g_{1}^{2}+225 \zeta(5)\left(g_{1}-g_{2}\right)\left(g_{1}+g_{2}\right)\left(3 g_{1}^{2}+g_{2}^{2}\right)+\pi^{6} g_{1}^{4}\right] \\
& +\frac{16}{135} \pi^{2} g_{1}^{4}\left[810 \pi^{4} \zeta(3)\left(g_{2}^{2}-g_{1}^{2}\right) g_{1}^{4}+900 \pi^{2} \zeta(5)\left(13 g_{1}^{4}-9 g_{2}^{2} g_{1}^{2}-4 g_{2}^{4}\right) g_{1}^{2}\right. \\
& +135\left(g_{1}^{2}-g_{2}^{2}\right)\left(36 \zeta(3)^{2}\left(2 g_{1}^{4}-g_{2}^{2} g_{1}^{2}+g_{2}^{4}\right)-35 \zeta(7)\left(8 g_{1}^{4}+5 g_{2}^{2} g_{1}^{2}+g_{2}^{4}\right)\right) \\
& \left.\left.+\pi^{8} g_{1}^{6}\right]+\cdots\right](b-1) \\
& +\left[2 \pi^{2} g_{1}^{2}+8 \pi^{4} g_{1}^{4}+\frac{4}{3} \pi^{2} g_{1}^{4}\left[66 \zeta(3)\left(g_{2}^{2}-g_{1}^{2}\right)-120 \zeta(5)\left(4 g_{1}^{2}+g_{2}^{2}\right)+5 \pi^{4} g_{1}^{2}\right]\right. \\
& +\frac{16}{45} \pi^{2} g_{1}^{4}\left[780 \pi^{2} \zeta(3)\left(g_{2}^{2}-g_{1}^{2}\right) g_{1}^{2}-75 \zeta(5)\left(\left(34 \pi^{2}-81\right) g_{1}^{4}+2\left(27+4 \pi^{2}\right) g_{2}^{2} g_{1}^{2}+27 g_{2}^{4}\right)\right. \\
& \left.+1575 \zeta(7)\left(15 g_{1}^{4}+4 g_{2}^{2} g_{1}^{2}+2 g_{2}^{4}\right)+7 \pi^{6} g_{1}^{4}\right]+\frac{8}{15} \pi^{2} g_{1}^{4}\left[-10 \pi^{4} g_{1}^{4}\left((53 \zeta(3)+88 \zeta(5)) g_{1}^{2}\right.\right. \\
& \left.+(20 \zeta(5)-53 \zeta(3)) g_{2}^{2}\right)+140 \pi^{2} g_{1}^{2}\left((65 \zeta(5)+166 \zeta(7)) g_{1}^{4}+45(\zeta(7)-\zeta(5)) g_{2}^{2} g_{1}^{2}\right. \\
& \left.+20(\zeta(7)-\zeta(5)) g_{2}^{4}\right)+15\left(8\left(57 \zeta(3)^{2}+480 \zeta(5) \zeta(3)-595 \zeta(7)-1806 \zeta(9)\right) g_{1}^{6}\right. \\
& -3\left(228 \zeta(3)^{2}+720 \zeta(5) \zeta(3)-595 \zeta(7)+840 \zeta(9)\right) g_{2}^{2} g_{1}^{4}+4(6 \zeta(3)(19 \zeta(3)-40 \zeta(5)) \\
& +595 \zeta(7)-840 \zeta(9)) g_{2}^{4} g_{1}^{2}-\left(228 \zeta(3)^{2}+720 \zeta(5) \zeta(3)-595 \zeta(7)\right. \\
& \left.\left.\left.840 \zeta(9)) g_{2}^{6}\right)+\pi^{8} g_{1}^{6}\right]+\ldots\right](b-1)^{2}+\mathcal{O}(b-1)^{3} \\
& +12)
\end{aligned}
$$

From (C.12) one can extract the effective coupling (4.16)ff.

\section{The strong coupling limit}

In this subsection, we want to make a strong coupling analysis of the saddle point equations (4.10) in the case in which $b=1$, i.e. on the sphere. In so doing, we will follow the same procedure as explained in the appendices of [43], with some additional details and complications. Before we begin in earnest with the study of the strong coupling limit, it is 
necessary to derive some integral identities. Of particular importance are (A.3) and (A.4). We define the function

$$
\theta(x):=x \operatorname{coth}(\pi x) .
$$

We use the same conventions for the Fourier transform as [43], i.e. $\hat{f}(\omega):=\int_{-\infty}^{\infty} d x e^{i x \omega} f(x)$. so that in the sense of distributions we get

$$
\hat{\theta}(\omega)=-\frac{1}{2 \sinh ^{2}\left(\frac{\omega}{2}\right)} .
$$

From equation (4.14) of [43], we take the following "formal" integral formula for $K(x)=$ $K_{v}(x ; 1)=K_{h}(x ; 1)$

$$
K(x)=f_{-\infty}^{\infty} \frac{\theta(w)}{x-w} d w .
$$

To make (D.3) correct, one needs to shift the argument of $K(x)$ by $z$ and average over $z$ such that the first two moments vanish. Specifically, we observe that

$$
\begin{aligned}
\int_{-\mu}^{\mu} d y \rho(y)(K(x-y)-K(x)) & =\int_{-\mu}^{\mu} d y \rho(y)\left(-y K^{\prime}(x)+\frac{1}{2} y^{2} K^{\prime \prime}(x)+\cdots\right) \\
& =-\mathfrak{m}_{1} K^{\prime}(x)+\frac{1}{2} \mathfrak{m}_{2} K^{\prime \prime}(x)+\cdots=\frac{1}{2} \mathfrak{m}_{2} K^{\prime \prime}(x)+\cdots
\end{aligned}
$$

so that (D.3) is applicable, i.e.

$$
\int_{-\mu}^{\mu} d y \rho(y)(K(x-y)-K(x))=f_{-\infty}^{\infty} \frac{d w}{x-w} \int_{-\mu}^{\mu} d y \rho(y)[\theta(w-y)-\theta(w)],
$$

which has been checked numerically. If we use (D.5) for the kernel $K$ as well as equation (A.4), we are able to show ${ }^{16}$

$$
\begin{aligned}
\frac{1}{\pi^{2}} & f_{-\mu}^{\mu} \frac{d y}{x-y} \sqrt{\frac{\mu^{2}-x^{2}}{\mu^{2}-y^{2}}} \int_{-\mu}^{\mu} d z \rho(z)[K(y-z)-K(y)] \\
& =-\frac{\sqrt{\mu^{2}-x^{2}}}{\pi^{2}} f_{-\infty}^{\infty} d w \int_{-\mu}^{\mu} d z \rho(z)(\theta(w-z)-\theta(w)) f_{-\mu}^{\mu} \frac{d y}{(x-y)(w-y)} \frac{1}{\sqrt{\mu^{2}-y^{2}}} \\
& =\theta(x)-(\rho \star \theta)(x)+\frac{1}{\pi} \int_{|w|>\mu} \frac{\operatorname{sgn}(\mathrm{w}) \mathrm{dw}}{w-x} \sqrt{\frac{\mu^{2}-x^{2}}{w^{2}-\mu^{2}}}[(\rho \star \theta)(w)-\theta(w)]
\end{aligned}
$$

where we have defined the convolution as $(\rho \star \theta)(x):=\int_{-\mu}^{\mu} d y \rho(y) \theta(x-y)$.

Having set up the necessary additional identities, we can start our analysis of the strong coupling behavior of the saddle point equations. We take the system of $r$ coupled integral equations for the cyclic quivers (4.10) for $b=1$ and using (A.5) we rewrite them as:

$$
\rho_{k}(x)=\frac{\sqrt{\mu_{k}^{2}-x^{2}}}{2 \pi g_{k}^{2}}-\frac{1}{\pi^{2}} \int_{-\mu_{k}}^{\mu_{k}} \frac{d y}{x-y} \sqrt{\frac{\mu_{k}^{2}-x^{2}}{\mu_{k}^{2}-y^{2}}} \sum_{l=1}^{r} \frac{\hat{\mathbf{a}}_{k l}}{2} \int_{-\mu_{l}}^{\mu_{l}} \rho_{l}(z)[K(y-z)-K(z)] d z,
$$

\footnotetext{
${ }^{16}$ The "sgn" part of the equation is a convention that can be absorbed in the definition of the square roots.
} 
where $\hat{\mathbf{a}}_{k l}:=2 \delta_{k l}-\delta_{k, l+1}-\delta_{k, l-1}$ is the SUr Cartan matrix and we have used $\sum_{l=1}^{r} \hat{\mathbf{a}}_{k l}=0$. Using (D.6), we arrive at

$$
\begin{array}{r}
\rho_{k}(x)-\sum_{l=1}^{r} \frac{\hat{\mathbf{a}}_{k l}}{2} \int_{-\mu_{l}}^{\mu_{l}} d y \rho_{l}(y)(\theta(x-y)-\theta(x))=\frac{\sqrt{\mu_{k}^{2}-x^{2}}}{2 \pi g_{k}^{2}} \\
-\frac{1}{\pi} \sum_{l=1}^{r} \frac{\hat{\mathbf{a}}_{k l}}{2} \int_{|w|>\mu_{k}} \frac{\operatorname{sgn}(w) d w}{w-x} \sqrt{\frac{\mu_{k}^{2}-x^{2}}{w^{2}-\mu_{k}^{2}}} \int_{-\mu_{l}}^{\mu_{l}} d y \rho_{l}(y)(\theta(w-y)-\theta(w))=: \mathbb{F}_{k}(x) .
\end{array}
$$

The functions $\mathbb{F}_{k}$ are the driving terms of the integral equations, i.e. our equations are written as

$$
\rho_{k}(x)-\sum_{l=1}^{r} \frac{\hat{\mathbf{a}}_{k l}}{2} \int_{-\mu_{l}}^{\mu_{l}} d y \rho_{l}(y)(\theta(x-y)-\theta(x))=\mathbb{F}_{k}(x) .
$$

In the strong coupling limit, the $\mathbb{F}_{k}(x)$ are dominated by their first term. Fourier transforming (D.9), using (D.2) and $\sum_{l=1}^{r} \hat{\mathbf{a}}_{k l}=0$, we get the set of equations

$$
\hat{\rho}_{k}(\omega)+\sum_{l=1}^{r} \frac{\hat{\mathbf{a}}_{k l}}{2} \frac{\hat{\rho}_{l}(\omega)}{2 \sinh ^{2} \frac{\omega}{2}}=\hat{\mathbb{F}}_{k}(\omega)+e^{-i \mu_{k} \omega} \hat{X}_{k}^{-}(\omega)+e^{i \mu_{k} \omega} \hat{X}_{k}^{+}(\omega),
$$

where the functions $\hat{X}_{k}^{ \pm}$are analytic in the upper/lower half plane respectively. Their presence is due to the fact that (D.9) only holds for $x \in\left(-\mu_{k}, \mu_{k}\right)$. We can now rewrite (D.10) as

$$
\sum_{l=1}^{r} \mathbb{A}_{k l}(\omega) \hat{\rho}_{l}(\omega)=\hat{\mathbb{F}}_{k}(\omega)+e^{-i \mu_{k} \omega} \hat{X}_{k}^{-}(\omega)+e^{i \mu_{k} \omega} \hat{X}_{k}^{+}(\omega)
$$

where

$$
\mathbb{A}_{k l}(\omega):=\frac{\left(e^{\omega}+e^{-\omega}\right) \delta_{k l}-\delta_{k, l-1}-\delta_{k, l+1}}{e^{\omega}+e^{-\omega}-2}
$$

with the indices $k, l$ subject to the identification $k \equiv k+r$ and $l \equiv l+r$. The implicit dependence on the number of gauge groups $r$ contained in many quantities, such as $\mathbb{A}_{k l}$, will not be explicitly noted in this appendix so as to not clutter the notation. Solving (D.11) for the densities by inverting the matrix $\mathbb{A}$, we get

$$
\begin{aligned}
\mathbb{K}(\omega) \hat{\rho}_{k}(\omega)= & \sum_{l=1}^{r}\left(\chi_{|k-l|-1}\left(e^{\omega}\right)+\chi_{r-1-|k-l|}\left(e^{\omega}\right)\right) \\
& \times\left(\hat{\mathbb{F}}_{l}(\omega)+e^{-i \mu_{l} \omega} \hat{X}_{l}^{-}(\omega)+e^{i \mu_{l} \omega} \hat{X}_{l}^{+}(\omega)\right),
\end{aligned}
$$

where $\chi_{j}(u):=\sum_{m=0}^{j} u^{j-2 m}$ are the characters of the $j+1$ dimensional representations of SU2 and we have defined the kernel

$$
\mathbb{K}(\omega):=\frac{e^{r \omega}-2+e^{-r \omega}}{e^{\omega}-2+e^{-\omega}}=\left(\frac{\sinh \frac{r \omega}{2}}{\sinh \frac{\omega}{2}}\right)^{2} .
$$

Using the well known formula $\sin (x)=\pi\left(\Gamma\left(\frac{x}{\pi}\right) \Gamma\left(1-\frac{x}{\pi}\right)\right)^{-1}$, we can express $\mathbb{K}(\omega)$ using the $\Gamma$ function as

$$
\mathbb{K}(\omega)=\frac{1}{\mathbb{G}_{+}(\omega) \mathbb{G}_{-}(\omega)}, \quad \text { where } \quad \mathbb{G}_{ \pm}(\omega):=\frac{1}{r}\left(\frac{\Gamma\left(1 \mp \frac{i r \omega}{2 \pi}\right)}{\Gamma\left(1 \mp \frac{i \omega}{2 \pi}\right)}\right)^{2},
$$


where the functions $\mathbb{G}_{ \pm}(\omega)$ have second order poles at $\mp i \nu_{n}$ with

$$
\nu_{n}=2 \pi \frac{n}{r}, \quad n=1,2,3, \ldots, \text { with } n \notin r \mathbb{N} .
$$

Expanding around the poles, we get

$$
\mathbb{G}_{ \pm}(\omega)=\frac{\alpha_{n}}{\left(\omega \pm i \nu_{n}\right)^{2}} \pm \frac{\beta_{n}}{\omega \pm i \nu_{n}}+\cdots
$$

where

$$
\alpha_{n}:=-\frac{4 \pi^{2}}{r^{3} \Gamma(n)^{2} \Gamma\left(1-\frac{n}{r}\right)^{2}}, \quad \beta_{n}:=\frac{4 \pi i\left(r \psi(n)-\psi\left(1-\frac{n}{r}\right)\right)}{r^{3} \Gamma(n)^{2} \Gamma\left(1-\frac{n}{r}\right)^{2}},
$$

and $\psi(x):=\Gamma^{\prime}(x) / \Gamma(x)$ is the digamma function. Observe that both $\alpha_{n}$ and $\beta_{n}$ go to zero very rapidly and that the $\beta_{n}$ are purely imaginary. We solve (D.13) by multiplying by $\mathbb{G}_{+}(\omega) e^{-i \mu_{k} \omega}$ and taking the negative frequency part. Ignoring to first approximation the $\hat{X}_{k}^{ \pm}$, we get

$$
\hat{\rho}_{k}(\omega)=\mathbb{G}_{-}(\omega) e^{i \mu_{k} \omega}\left[\mathbb{G}_{+}(\omega) e^{-i \mu_{k} \omega} \sum_{l=1}^{r} \mathbb{B}_{k l}(\omega) \hat{\mathbb{F}}_{l}(\omega)\right]_{-},
$$

where we used the matrix $\mathbb{B}$ with

$$
\mathbb{B}_{k l}(\omega):=\chi_{|k-l|-1}\left(e^{\omega}\right)+\chi_{r-1-|k-l|}\left(e^{\omega}\right)
$$

and the definition

$$
\mathcal{F}_{ \pm}(\omega):= \pm \lim _{\epsilon \rightarrow 0+} \int_{-\infty}^{\infty} \frac{d \omega^{\prime}}{2 \pi i} \frac{\mathcal{F}\left(\omega^{\prime}\right)}{\omega^{\prime}-\omega \mp i \epsilon} .
$$

The functions $\mathcal{F}_{ \pm}(\omega)$ are analytic in the upper/lower half-planes and the integral contours are to be closed in the upper/lower half-plane. The contour integral of (D.19) will give us the residues of $\mathbb{G}_{+}(\omega) \mathbb{B}_{k l}(\omega)$ at $-i \nu_{n}$. Expanding, we find

$$
\operatorname{Res}\left(\mathbb{G}_{+}(\omega) \mathbb{B}_{k l}(\omega),-i \nu_{n}\right)=\alpha_{n}\left(\partial_{\omega} \mathbb{B}_{k l}\right)\left(-i \nu_{n}\right)+\beta_{n} \mathbb{B}_{k l}\left(-i \nu_{n}\right) .
$$

Through brute force, we discover that

$$
\mathbb{B}_{k l}\left(-i \nu_{n}\right)= \begin{cases}r & \text { for } n \in r \mathbb{N} \\ (-1)^{k+l-1} r & \text { for } n \in \frac{r}{2} \mathbb{N} \\ 0 & \text { otherwise }\end{cases}
$$

as well as

$$
\left(\partial_{\omega} \mathbb{B}_{k l}\right)\left(-i \nu_{n}\right)= \begin{cases}0 & \text { for } n \in r \mathbb{N} \\ 0 & \text { for } n \in \frac{r}{2} \mathbb{N} \\ \frac{i r \cos \left(|k-l| \nu_{n}\right)}{\sin \left(\nu_{n}\right)} & \text { otherwise }\end{cases}
$$

Using the above, we find the residue of the poles $-i \nu_{n}$ :

$$
\mathbb{R}_{k l}(n):=\operatorname{Res}\left(\mathbb{G}_{+}(\omega) \mathbb{B}_{k l}(\omega),-i \nu_{n}\right)= \begin{cases}0 & \text { for } n \in r \mathbb{N}, \\ \beta_{n}(-1)^{k+l-1} r & \text { for } n \in \frac{r}{2} \mathbb{N} \\ \alpha_{n} \frac{i r \cos \left(|k-l| \nu_{n}\right)}{\sin \left(\nu_{n}\right)} & \text { otherwise }\end{cases}
$$


We observe experimentally that the matrices $\mathbb{R}_{k l}(n)$ all commute, that the vector $(1,1$, $\ldots, 1)^{t}$ is the only common eigenvector of $\mathbb{R}_{k l}(n)$ and that it has eigenvalue zero. Plugging (D.21) into (D.19) we get

$$
\hat{\rho}_{k}(\omega)=\frac{1}{\mathbb{K}(\omega)} \sum_{l=1}^{r} \mathbb{B}_{k l}(\omega) \hat{\mathbb{F}}_{l}(\omega)-\mathbb{G}_{-}(\omega) e^{i \mu_{k} \omega} \sum_{n=1}^{\infty} \frac{e^{-\mu_{k} \nu_{n}}}{\omega+i \nu_{n}} \sum_{l=1}^{r} \mathbb{R}_{k l}(n) \hat{\mathbb{F}}_{l}\left(-i \nu_{n}\right),
$$

where we have to use (D.25) for the residues. Observe that, contrary to what one might think at first glance, (D.26) is indeed analytic in the lower half plane since the poles at $-i \nu_{n}$ cancel.

We now need to normalize the densities. Since the Fourier transform of (D.26) will represent the density $\rho_{k}(x)$ well only for positive $x$, we use the fact that the densities should be symmetric and demand

$$
1=2 \int_{0}^{\mu_{k}} d x \rho_{k}(x)=\lim _{\epsilon \rightarrow 0+} \int_{-\infty}^{\infty} \frac{d \omega}{\pi i} \frac{\hat{\rho}_{k}(\omega)}{\omega-i \epsilon}=2\left(\hat{\rho}_{k}\right)_{+}(0),
$$

where we used (D.21). Thus, we need to close the contour in the upper half plane.

Taking the residues and using $\frac{1}{\omega \mp i \epsilon}=$ p.v. $\pm i \pi \delta$ as well as the symmetry under $\omega \rightarrow-\omega$ of $\mathbb{B}, \mathbb{K}$ and $\hat{\mathbb{F}}_{k}$ gives

$$
\begin{aligned}
1= & \frac{1}{\mathbb{K}(0)} \sum_{l=1}^{r} \mathbb{B}_{k l}(0) \hat{\mathbb{F}}_{l}(0)+2 i \mathbb{G}_{-}(0) \sum_{n=1}^{\infty} \frac{e^{-\mu_{k} \nu_{n}}}{\nu_{n}} \sum_{l=1}^{r} \mathbb{R}_{k l}(n) \hat{\mathbb{F}}_{l}\left(-i \nu_{n}\right) \\
& -2 \sum_{m, n=1}^{\infty} \frac{e^{-\mu_{k}\left(\nu_{m}+\nu_{n}\right)}}{\nu_{m}\left(\nu_{m}+\nu_{n}\right)}\left(\beta_{m}-i \alpha_{m} \frac{\nu_{n}+2 \nu_{m}+\nu_{n} \nu_{m} \mu_{k}+\nu_{m}^{2} \mu_{k}}{\nu_{m}\left(\nu_{m}+\nu_{n}\right)}\right) \sum_{l=1}^{r} \mathbb{R}_{k l}(n) \hat{\mathbb{F}}_{l}\left(-i \nu_{n}\right)
\end{aligned}
$$

Using $\mathbb{K}(0)=r^{2}, \mathbb{B}_{k l}(0)=r$ and $\mathbb{G}_{-}(0)=\frac{1}{r}$, we get

$$
\begin{aligned}
1= & \frac{1}{r} \sum_{l=1}^{r} \hat{\mathbb{F}}_{l}(0)+\frac{2 i}{r} \sum_{n=1}^{\infty} \frac{e^{-\mu_{k} \nu_{n}}}{\nu_{n}} \sum_{l=1}^{r} \mathbb{R}_{k l}(n) \hat{\mathbb{F}}_{l}\left(-i \nu_{n}\right) \\
& -2 \sum_{m, n=1}^{\infty} \frac{e^{-\mu_{k}\left(\nu_{m}+\nu_{n}\right)}}{\nu_{m}\left(\nu_{m}+\nu_{n}\right)}\left(\beta_{m}-i \alpha_{m} \frac{\nu_{n}+2 \nu_{m}+\nu_{n} \nu_{m} \mu_{k}+\nu_{m}^{2} \mu_{k}}{\nu_{m}\left(\nu_{m}+\nu_{n}\right)}\right) \sum_{l=1}^{r} \mathbb{R}_{k l}(n) \hat{\mathbb{F}}_{l}\left(-i \nu_{n}\right) .
\end{aligned}
$$

The terms in the second line of (D.26) are exponentially suppressed. We will now make an assumption that is justified by the self-consistency of the results. As in [43], in the large coupling limit, the driving terms defined in (D.8) are dominated by their first term, so that

$$
\mathbb{F}_{k}(x)=\frac{1}{2 \pi g_{k}^{2}} \sqrt{\mu_{k}^{2}-x^{2}} \Longrightarrow \hat{\mathbb{F}}_{k}(\omega)=\frac{\mu_{k} J_{1}\left(\mu_{k} \omega\right)}{2 g_{k}^{2} \omega}, \quad \hat{\mathbb{F}}_{k}(0)=\frac{\mu_{k}^{2}}{4 g_{k}^{2}}, \quad \hat{\mathbb{F}}_{k}\left(-i \nu_{n}\right)=\frac{e^{\mu_{k} \nu_{n}}}{g_{k}^{2}} \sqrt{\frac{\mu_{k}}{8 \pi \nu_{n}^{3}}},
$$

where $J_{n}$ are the Bessel functions of the first kind. Using this approximation for the driving terms and dropping the exponentially suppressed terms in (D.29), explicit numerical solutions show that for large values of the couplings, the widths of the densities $\mu_{k}$ are very 
close to being equal to each other (as long as the ratios of the couplings $g_{k} / g_{l}$ is roughly of order one) and are to a very good approximation given by

$$
\mu_{k}=\bar{\mu}:=2 \sqrt{r\left(\sum_{k=1}^{r} g_{k}^{-2}\right)^{-1}}=2 \sqrt{\frac{r g_{1}^{2} \cdots g_{r}^{2}}{\sum_{i=1}^{r} \prod_{k \neq i} g_{k}^{2}}} \quad \forall k .
$$

It can be seen that this is a solution of (D.29) if we ignore the (numerically suppressed) pieces containing $\hat{\mathbb{F}}_{k}\left(-i \nu_{n}\right)$, which implies

$$
1=\frac{1}{\mathbb{K}(0)} \sum_{l=1}^{r} \mathbb{B}_{k l}(0) \hat{\mathbb{F}}_{l}(0)=\frac{1}{r^{2}} \sum_{l=1}^{r} r \frac{\mu_{l}^{2}}{4 g_{l}^{2}}=\frac{1}{4 r} \sum_{l=1}^{r} \frac{\mu_{l}^{2}}{g_{l}^{2}} .
$$

An a posteriori justification for neglecting the $\hat{\mathbb{F}}_{k}\left(-i \nu_{n}\right)$ is that, for $g_{k}$ that are not wildly different, see (4.13), the $\hat{\mathbb{F}}_{k}$ will be roughly equal, so that the term $\sum_{l=1}^{r} \mathbb{R}_{k l}(n) \hat{\mathbb{F}}_{l}\left(-i \nu_{n}\right)$ will be small since $(1,1, \ldots, 1)$ is an eigenvector of the $\mathbb{R}$ matrices with eigenvalue zero.

The densities themselves have in the strong coupling limit the same shape as the $\mathcal{N}=4$ one. The leading behavior of the Wilson loop expectation values at large values of the coupling is hence (using the asymptotic expression $I_{1}(x) \sim e^{x} / \sqrt{2 \pi x}$ )

$$
\left\langle W_{k}^{ \pm}\right\rangle=\hat{\rho}_{k}\left(2 \pi i b^{ \pm 1}\right) \sim \frac{e^{2 \pi b^{ \pm 1} \bar{\mu}}}{2 \pi^{2} b^{ \pm 3 / 2} \bar{\mu}^{\frac{3}{2}}}+\mathcal{O}(b-1)^{2} .
$$

Due to the exponential term, the strong coupling limit of the effective couplings is simply given by comparing (D.31) with the width $\mu$ for $\mathcal{N}=4$. Since for $\mathcal{N}=4$ SYM we have $\mu^{2}=4 g^{2}$, comparing with (D.31) leads to equation (4.24) in the main text. In particular, the strong coupling limit of the Bremsstrahlung functions is

$$
B_{k}=\frac{\mu_{k}}{2 \pi}-\frac{3}{8 \pi^{2}}
$$

A more detailed analysis of (D.29) should also allow one to extract the leading corrections to the relation (4.24).

Open Access. This article is distributed under the terms of the Creative Commons Attribution License (CC-BY 4.0), which permits any use, distribution and reproduction in any medium, provided the original author(s) and source are credited.

\section{References}

[1] E. Pomoni, Integrability in $N=2$ superconformal gauge theories, Nucl. Phys. B 893 (2015) 21 [arXiv:1310.5709] [InSPIRE].

[2] V. Mitev and E. Pomoni, Exact effective couplings of four dimensional gauge theories with $\mathcal{N}=2$ supersymmetry, Phys. Rev. D 92 (2015) 125034 [arXiv:1406.3629] [INSPIRE].

[3] N. Beisert et al., Review of AdS/CFT Integrability: An Overview, Lett. Math. Phys. 99 (2012) 3 [arXiv: 1012.3982] [INSPIRE].

[4] V. Pestun, Localization of gauge theory on a four-sphere and supersymmetric Wilson loops, Commun. Math. Phys. 313 (2012) 71 [arXiv:0712.2824] [INSPIRE]. 
[5] J. Teschner, Exact results on $\mathcal{N}=2$ supersymmetric gauge theories, arXiv:1412.7145.

[6] V. Pestun, Localization of the four-dimensional $\mathcal{N}=4 S Y M$ to a two-sphere and $1 / 8$ BPS Wilson loops, JHEP 12 (2012) 067 [arXiv:0906.0638] [INSPIRE].

[7] J. Gomis, T. Okuda and V. Pestun, Exact Results for 't Hooft Loops in Gauge Theories on $S^{4}$, JHEP 05 (2012) 141 [arXiv: 1105.2568] [INSPIRE].

[8] A. Lewkowycz and J. Maldacena, Exact results for the entanglement entropy and the energy radiated by a quark, JHEP 05 (2014) 025 [arXiv: 1312.5682] [INSPIRE].

[9] N. Drukker and V. Forini, Generalized quark-antiquark potential at weak and strong coupling, JHEP 06 (2011) 131 [arXiv:1105.5144] [INSPIRE].

[10] D. Correa, J. Maldacena and A. Sever, The quark anti-quark potential and the cusp anomalous dimension from a TBA equation, JHEP 08 (2012) 134 [arXiv:1203.1913] [INSPIRE].

[11] N. Drukker, Integrable Wilson loops, JHEP 10 (2013) 135 [arXiv:1203.1617] [InSPIRE].

[12] E. Gerchkovitz, J. Gomis and Z. Komargodski, Sphere Partition Functions and the Zamolodchikov Metric, JHEP 11 (2014) 001 [arXiv:1405.7271] [INSPIRE].

[13] J. Gomis, P.-S. Hsin, Z. Komargodski, A. Schwimmer, N. Seiberg and S. Theisen, Anomalies, Conformal Manifolds and Spheres, JHEP 03 (2016) 022 [arXiv: 1509.08511] [INSPIRE].

[14] N. Hama and K. Hosomichi, Seiberg-Witten Theories on Ellipsoids, JHEP 09 (2012) 033 [arXiv:1206.6359] [INSPIRE].

[15] L. Bao, V. Mitev, E. Pomoni, M. Taki and F. Yagi, Non-Lagrangian Theories from Brane Junctions, JHEP 01 (2014) 175 [arXiv:1310.3841] [INSPIRE].

[16] H. Hayashi, H.-C. Kim and T. Nishinaka, Topological strings and $5 d T_{N}$ partition functions, JHEP 06 (2014) 014 [arXiv: 1310.3854] [InSPIRE].

[17] V. Mitev and E. Pomoni, Toda 3-Point Functions From Topological Strings, JHEP 06 (2015) 049 [arXiv: 1409.6313] [INSPIRE].

[18] M. Isachenkov, V. Mitev and E. Pomoni, Toda 3-Point Functions From Topological Strings II, arXiv:1412.3395 [INSPIRE].

[19] B. Fiol, E. Gerchkovitz and Z. Komargodski, Exact Bremsstrahlung Function in $\mathcal{N}=2$ Superconformal Field Theories, Phys. Rev. Lett. 116 (2016) 081601 [arXiv:1510.01332] [INSPIRE].

[20] CTEQ collaboration, R. Brock et al., Handbook of perturbative QCD: Version 1.0, Rev. Mod. Phys. 67 (1995) 157 [InSPIRE].

[21] D. Correa, J. Henn, J. Maldacena and A. Sever, An exact formula for the radiation of a moving quark in $\mathcal{N}=4$ super Yang-Mills, JHEP 06 (2012) 048 [arXiv:1202.4455] [INSPIRE].

[22] A.M. Polyakov, Gauge Fields as Rings of Glue, Nucl. Phys. B 164 (1980) 171 [InSPIRE].

[23] S.S. Gubser, I.R. Klebanov and A.M. Polyakov, A Semiclassical limit of the gauge/string correspondence, Nucl. Phys. B 636 (2002) 99 [hep-th/0204051] [INSPIRE].

[24] D. Correa, J. Henn, J. Maldacena and A. Sever, The cusp anomalous dimension at three loops and beyond, JHEP 05 (2012) 098 [arXiv:1203.1019] [INSPIRE]. 
[25] J.M. Henn and T. Huber, Systematics of the cusp anomalous dimension, JHEP 11 (2012) 058 [arXiv:1207.2161] [INSPIRE].

[26] J.M. Henn and T. Huber, The four-loop cusp anomalous dimension in $\mathcal{N}=4$ super Yang-Mills and analytic integration techniques for Wilson line integrals, JHEP 09 (2013) 147 [arXiv: 1304.6418] [INSPIRE].

[27] N. Beisert, B. Eden and M. Staudacher, Transcendentality and Crossing, J. Stat. Mech. 0701 (2007) P01021 [hep-th/0610251] [INSPIRE].

[28] L. Freyhult, Review of AdS/CFT Integrability, Chapter III.4: Twist States and the cusp Anomalous Dimension, Lett. Math. Phys. 99 (2012) 255 [arXiv: 1012.3993] [InSPIRE].

[29] D. Dorigoni and Y. Hatsuda, Resurgence of the Cusp Anomalous Dimension, JHEP 09 (2015) 138 [arXiv: 1506.03763] [INSPIRE].

[30] I. Aniceto, The Resurgence of the Cusp Anomalous Dimension, J. Phys. A 49 (2016) 065403 [arXiv: 1506.03388] [INSPIRE].

[31] G.P. Korchemsky, Review of AdS/CFT Integrability, Chapter IV.4: Integrability in QCD and $N<4$ SYM, Lett. Math. Phys. 99 (2012) 425 [arXiv:1012.4000] [InSPIRE].

[32] N. Beisert, G. Ferretti, R. Heise and K. Zarembo, One-loop QCD spin chain and its spectrum, Nucl. Phys. B 717 (2005) 137 [hep-th/0412029] [INSPIRE].

[33] A. Gadde, E. Pomoni and L. Rastelli, The Veneziano Limit of $\mathcal{N}=2$ Superconformal QCD: Towards the String Dual of $\mathcal{N}=2 \mathrm{SU}\left(N_{c}\right) S Y M$ with $N_{f}=2 N_{c}$, arXiv:0912.4918 [INSPIRE].

[34] A. Gadde, E. Pomoni and L. Rastelli, Spin Chains in $\mathcal{N}=2$ Superconformal Theories: From the $Z_{2}$ Quiver to Superconformal QCD, JHEP 06 (2012) 107 [arXiv:1006.0015] [INSPIRE].

[35] A. Gadde and L. Rastelli, Twisted Magnons, JHEP 04 (2012) 053 [arXiv:1012.2097] [INSPIRE].

[36] E. Pomoni and C. Sieg, From $\mathcal{N}=4$ gauge theory to $\mathcal{N}=2$ conformal QCD: three-loop mixing of scalar composite operators, arXiv:1105.3487 [INSPIRE].

[37] P. Liendo, E. Pomoni and L. Rastelli, The Complete One-Loop Dilation Operator of $\mathcal{N}=2$ SuperConformal QCD, JHEP 07 (2012) 003 [arXiv:1105.3972] [INSPIRE].

[38] A. Gadde, P. Liendo, L. Rastelli and W. Yan, On the Integrability of Planar $\mathcal{N}=2$ Superconformal Gauge Theories, JHEP 08 (2013) 015 [arXiv: 1211.0271] [INSPIRE].

[39] M. Bershadsky, Z. Kakushadze and C. Vafa, String expansion as large- $N$ expansion of gauge theories, Nucl. Phys. B 523 (1998) 59 [hep-th/9803076] [INSPIRE].

[40] M. Bershadsky and A. Johansen, Large- $N$ limit of orbifold field theories, Nucl. Phys. B 536 (1998) 141 [hep-th/9803249] [INSPIRE].

[41] A.E. Lawrence, N. Nekrasov and C. Vafa, On conformal field theories in four-dimensions, Nucl. Phys. B 533 (1998) 199 [hep-th/9803015] [INSPIRE].

[42] O. Aharony, M. Berkooz and S.-J. Rey, Rigid holography and six-dimensional $\mathcal{N}=(2,0)$ theories on $A d S_{5} \times \mathbb{S}^{1}, J H E P 03$ (2015) 121 [arXiv: 1501.02904] [INSPIRE].

[43] F. Passerini and K. Zarembo, Wilson Loops in $\mathcal{N}=2$ super-Yang-Mills from Matrix Model, JHEP 09 (2011) 102 [Erratum ibid. 1110 (2011) 065] [arXiv:1106.5763] [INSPIRE]. 
[44] J.G. Russo and K. Zarembo, Large- $N$ Limit of $\mathcal{N}=2 \mathrm{SU}(N)$ Gauge Theories from Localization, JHEP 10 (2012) 082 [arXiv:1207.3806] [INSPIRE].

[45] J.G. Russo and K. Zarembo, Massive $\mathcal{N}=2$ Gauge Theories at Large- $N$, JHEP 11 (2013) 130 [arXiv: 1309.1004] [INSPIRE].

[46] J.G. Russo and K. Zarembo, Localization at Large- $N$, in proceedings of 100 th anniversary of the birth of I.Ya. Pomeranchuk, Moscow, Russia, 5-6 Jun 2013 [arXiv:1312.1214] [INSPIRE].

[47] D. Marmiroli, Phase structure of $\mathcal{N}=2^{*} S Y M$ on ellipsoids, arXiv:1410.4715 [INSPIRE].

[48] P.A. Baikov and K.G. Chetyrkin, Four Loop Massless Propagators: An Algebraic Evaluation of All Master Integrals, Nucl. Phys. B 837 (2010) 186 [arXiv:1004.1153] [INSPIRE].

[49] P.S. Howe, K.S. Stelle and P.C. West, A Class of Finite Four-Dimensional Supersymmetric Field Theories, Phys. Lett. B 124 (1983) 55 [INSPIRE].

[50] J.C. Collins, Renormalization. An Introduction To Renormalization, The Renormalization Group, And The Operator Product Expansion, Cambridge University Press (1986).

[51] A.G. Grozin, Lectures on multiloop calculations, Int. J. Mod. Phys. A 19 (2004) 473 [hep-ph/0307297] [INSPIRE].

[52] A.V. Manohar and M.B. Wise, Heavy quark physics, Cambridge University Press (2007).

[53] K.G. Chetyrkin, J.H. Kuhn and M. Steinhauser, RunDec: A Mathematica package for running and decoupling of the strong coupling and quark masses, Comput. Phys. Commun. 133 (2000) 43 [hep-ph/0004189] [INSPIRE].

[54] B. Fraser, Higher rank Wilson loops in the $\mathcal{N}=2 \mathrm{SU}(N) \times \mathrm{SU}(N)$ conformal quiver, J. Phys. A 49 (2016) 02LT03 [arXiv:1503.05634] [InSPIRE].

[55] A. Grozin, J.M. Henn, G.P. Korchemsky and P. Marquard, Three Loop Cusp Anomalous Dimension in QCD, Phys. Rev. Lett. 114 (2015) 062006 [arXiv:1409.0023] [INSPIRE].

[56] A. Grozin, J.M. Henn, G.P. Korchemsky and P. Marquard, The three-loop cusp anomalous dimension in QCD and its supersymmetric extensions, JHEP 01 (2016) 140 [arXiv: 1510.07803] [INSPIRE].

[57] G.P. Korchemsky, Asymptotics of the Altarelli-Parisi-Lipatov Evolution Kernels of Parton Distributions, Mod. Phys. Lett. A 4 (1989) 1257 [InSPIRE].

[58] G.P. Korchemsky and G. Marchesini, Structure function for large $x$ and renormalization of Wilson loop, Nucl. Phys. B 406 (1993) 225 [hep-ph/9210281] [INSPIRE].

[59] S. Caron-Huot, Notes on the scattering amplitude/Wilson loop duality, JHEP 07 (2011) 058 [arXiv: 1010.1167] [INSPIRE].

[60] M. Leoni, A. Mauri and A. Santambrogio, Four-point amplitudes in $\mathcal{N}=2 S C Q C D$, JHEP 09 (2014) 017 [Erratum ibid. 1502 (2015) 022] [arXiv: 1406.7283] [INSPIRE].

[61] M. Leoni, A. Mauri and A. Santambrogio, On the amplitude/Wilson loop duality in $\mathcal{N}=2$ SCQCD, Phys. Lett. B 747 (2015) 325 [arXiv:1502.07614] [InSPIRE].

[62] N. Gromov and A. Sever, Analytic Solution of Bremsstrahlung TBA, JHEP 11 (2012) 075 [arXiv: 1207.5489] [INSPIRE].

[63] N. Gromov, F. Levkovich-Maslyuk and G. Sizov, Analytic Solution of Bremsstrahlung TBA II: Turning on the Sphere Angle, JHEP 10 (2013) 036 [arXiv:1305.1944] [INSPIRE]. 
[64] G. Sizov and S. Valatka, Algebraic Curve for a Cusped Wilson Line, JHEP 05 (2014) 149 [arXiv:1306.2527] [INSPIRE].

[65] R.A. Janik, Review of AdS/CFT Integrability, Chapter III.5: Lúscher Corrections, Lett. Math. Phys. 99 (2012) 277 [arXiv:1012.3994] [INSPIRE].

[66] K. Papadodimas, Topological Anti-Topological Fusion in Four-Dimensional Superconformal Field Theories, JHEP 08 (2010) 118 [arXiv:0910.4963] [INSPIRE].

[67] M. Baggio, V. Niarchos and K. Papadodimas, Exact correlation functions in $\mathrm{SU}(2) \mathcal{N}=2$ superconformal QCD, Phys. Rev. Lett. 113 (2014) 251601 [arXiv:1409.4217] [INSPIRE].

[68] M. Baggio, V. Niarchos and K. Papadodimas, $t t^{*}$ equations, localization and exact chiral rings in $4 d \mathcal{N}=2$ SCFTs, JHEP 02 (2015) 122 [arXiv:1409.4212] [INSPIRE].

[69] M. Baggio, V. Niarchos and K. Papadodimas, On exact correlation functions in $\mathrm{SU}(N)$ $\mathcal{N}=2$ superconformal QCD, JHEP 11 (2015) 198 [arXiv:1508.03077] [INSPIRE].

[70] N. Gromov and G. Sizov, Exact Slope and Interpolating Functions in $\mathcal{N}=6$ Supersymmetric Chern-Simons Theory, Phys. Rev. Lett. 113 (2014) 121601 [arXiv:1403.1894] [InSPIRE].

[71] L.F. Alday and J.M. Maldacena, Comments on operators with large spin, JHEP 11 (2007) 019 [arXiv: 0708.0672] [INSPIRE].

[72] B. Fiol, B. Garolera and G. Torrents, Probing $\mathcal{N}=2$ superconformal field theories with localization, JHEP 01 (2016) 168 [arXiv:1511.00616] [INSPIRE]. 